\title{
ON THE EXISTENCE AND EXPLICIT REPRESENTABILITY OF STRONG SOLUTIONS OF LÉVY NOISE DRIVEN SDE'S WITH IRREGULAR COEFFICIENTS*
}

\author{
THILO MEYER-BRANDIS ${ }^{\dagger}$ AND FRANK PROSKE ${ }^{\ddagger}$
}

\begin{abstract}
We give a method to represent strong solutions of stochastic differential equations driven by Lévy processes, explicitly. Furthermore we employ these explicit representations to study strong solutions of a certain class of SDE's, whose coefficients are not necessarily Lipschitz continuous.
\end{abstract}

Key words. Lévy processes, stochastic differential equations, white noise analysis.

1991 MSC Subject Classifications. 60G51, 60G35, 60H15, 60H40, 60H15, 91B70

\section{Introduction}

In recent years stochastic differential equations (SDE's) for Lévy processes have become of much current interest for applications to mathematical finance, neurobiology and other areas of natural sciences. In this paper we want to demonstrate how white noise concepts for Lévy processes can be effectively used to solve a fully non-linear problem. More precisely, we present a method which enables us to determine solutions of SDE's driven by Lévy processes, explicitly. Moreover, this approach provides criteria for the existence of strong solutions of a certain class of SDE's whose coefficients are not Lipschitz continuous. To find explicit solutions is usually a challenging and difficult task of both theoretical and practical significance. We are convinced that our method grants new insights into the nature of solutions of LévyItô SDE's. In addition, it exhibits potential to yield dividends in other important applications.

In [15] the authors apply concepts from Gaussian white noise analysis to represent solutions of SDE's driven by a Brownian motion. Consider the 1-dimensional Itôdiffusion

$$
d Y_{t}=b\left(Y_{t}\right) d t+\sigma\left(Y_{t}\right) d B_{t}, Y_{0}=y, 0 \leq t \leq T,
$$

where $b$ is the drift, $\sigma$ the diffusion coefficient and $B_{t}$ the Brownian motion. It is proven in [15] that under certain conditions on $b$ and $\sigma$ a (global) strong solution $Y_{t}$ of the SDE takes the explicit form

$$
Y_{t}=E_{\widehat{\mu}}\left[u\left(\widehat{B}_{t}\right) M_{T}^{\diamond}\right]
$$

where

$$
\begin{aligned}
M_{T}^{\diamond}= & \exp ^{\diamond}\left\{\int_{0}^{T}\left(W_{t}+\frac{b\left(u\left(\widehat{B}_{t}\right)\right)}{\sigma\left(u\left(\widehat{B}_{t}\right)\right)}-\frac{1}{2} \sigma^{\prime}\left(u\left(\widehat{B}_{t}\right)\right)\right) d \widehat{B}_{t}\right. \\
& \left.-\frac{1}{2} \int_{0}^{T}\left(W_{t}+\frac{b\left(u\left(\widehat{B}_{t}\right)\right)}{\sigma\left(u\left(\widehat{B}_{t}\right)\right)}-\frac{1}{2} \sigma^{\prime}\left(u\left(\widehat{B}_{t}\right)\right)\right) d t\right\}
\end{aligned}
$$

\footnotetext{
*Received: October 29, 2005; accepted: January 6, 2006. Communicated by Shi Jin.

${ }^{\dagger}$ Centre of Mathematics for Applications (CMA), Department of Mathematics, University of Oslo, P. O. Box 1053 Blindern, N-0316 Oslo, Norway (meyerbr@math.uio.no).

$\ddagger$ Centre of Mathematics for Applications (CMA), Department of Mathematics, University of Oslo, P. O. Box 1053 Blindern, N-0316 Oslo, Norway (proske@math.uio.no).
} 
and where $u$ is a solution of the ordinary differential equation

$$
u^{\prime}=\sigma(u), u(0)=y .
$$

Here $W_{s}=W_{s}(\omega)$ is the (singular) white noise and $\diamond$ is the Wick product with respect to the white noise probability space $(\Omega, \mathcal{F}, \mu)$. The process $\widehat{B}_{t}=\widehat{B}_{t}(\widehat{\omega})$ is an auxiliary Brownian motion on the probability space $(\widehat{\Omega}, \widehat{\mathcal{F}}, \widehat{\mu})$, which is a copy of the initial white noise space. The formula involves (stochastic) Bochner integrals on the Hida distribution space $(\mathcal{S})^{*}$.

Using Lévy white noise theory, we adapt these ideas in this paper to Lévy-Itô diffusions, i.e. jump diffusions of the type

$$
d Y_{t}=b\left(Y_{t-}\right) d t+\sigma\left(Y_{t-}\right) d B_{t}+\gamma\left(Y_{t-}\right) d L_{t}, Y_{0}=y, 0 \leq t \leq T,
$$

where $b, \sigma, \gamma: \mathbb{R} \longrightarrow \mathbb{R}$ are measurable functions, $B_{t}$ a Brownian motion and $L_{t}$ a pure jump Lévy process with Lévy measure $\nu(d x)$.

As in the purely Gaussian case we deduce under certain conditions on $b, \sigma, \gamma$ and $\nu$ a general solution formula for this SDE. To the best of our knowledge, results about explicit strong solutions of SDE's are only in existence for continuous processes as driving noise. For example, [33] obtain explicit chaos expansions of strong solution's of SDE's driven by a Brownian motion. However, the kernels of the expansions must be determined as solutions of systems of ODE's or PDE's. Another approach is due to [4], [28] who construct strong solutions of SDE's driven by Brownian motion from solutions of ODE's, pathwisely. However, these methods reveal the deficiency to fail, if the coefficients of the SDE are not regular enough.

In the second part of the paper we mainly focus on SDE's driven by Brownian motion and give two first applications of the explicit representation. In Section 4 we state explicit chaos expansions of strong solutions. In Section 5 we establish an existence and uniqueness result for strong solutions of SDE's with irregular drift coefficients, i.e. with coefficients, which are e.g. not necessarily Lipschitz continuous, Sobolev differentiable or continuous. In this context we also formulate a convergence Theorem and a comparison Theorem for SDE's with irregular coefficients. We establish an $L^{2}$-integrability criterion on the drift $b$ to guarantee existence of unique strong solutions of distorted Brownian motions. To the best of our knowledge, this is the weakest condition known to ensure strong solutions of distorted Brownian motions.

The study of SDE's with less "nicely" behaved coefficients is important, since they arise in a broad range of stochastic control problems. Strong solutions of Brownian motion driven SDE's with non-Lipschitzian coefficients are treated e.g. by [30], [31], [32], [35], [36], [6], [7], [14]. Recently, new ideas were developed in [5]. In the current literature one finds a scarce number of results pertaining to strong solutions of LévyItô diffusions with irregular coefficients. Let us mention that [1] derives a condition in the case of symmetric stable processes of order $\alpha>1$, which guarantees strong solutions and pathwise uniqueness. This condition can be regarded as an analogue of the Yamada-Watanabe condition for the Brownian motion case, which presumes continuity on the coefficient $\gamma$. Other results on this topic can be e.g. found in [34] and [2]. With this paper we aim at contributing to a better understanding of Lévy-Itô diffusions.

The paper is organized as follows: In Section 2 we recall some concepts from white noise theory for Lévy processes, developed in [17], [18] and [23]. In Section 3 , for convenience of transparency we first focus on pure jump Lévy processes and 
derive a general solution formula for SDE's in this case. Then we turn to the general case. Section 4 deals with explicit chaos expansions of strong solutions. Section 5 is devoted to the existence and uniqueness of strong solutions of SDE's with irregular drift coefficients.

\section{White noise framework}

In this Section we provide a brief review of some concepts of a white noise theory for Lévy processes, developed in [18] and [23]. For general information about white noise theory the reader is referred to the excellent accounts of [8], [11] and [20].

Let us recall that a Lévy process $L(t)$ is a stochastic process on $\mathbb{R}_{+}$, which has independent and stationary increments starting at zero, i.e. $L(0)=0$. The process $L(t)$ is by its nature a càdlàg semimartingale, which is uniquely determined by the characteristic triplet

$$
\left(B_{t}, C_{t}, \hat{\mu}\right)=(a \cdot t, \sigma \cdot t, d t \nu(d x))
$$

where $a, \sigma$ are constants and where $\nu$ is the Lévy measure on $\mathbb{R}_{0}:=\mathbb{R}-\{0\}$. We denote by $\pi$ the product measure $\pi(d t, d x):=d t \nu(d x)$. For more information about Lévy processes consult e.g. [3], [27] or [10].

To avoid unnecessary technical complications we first recapitulate our white noise framework in the case of pure jump Lévy processes, that is we consider Lévy processes in (2.1) with $a=\sigma=0$. At the end of this Section we shortly explain the extension of the pure jump setting to the general case.

In the following we denote by $\mathcal{S}\left(\mathbb{R}^{d}\right)$ the Schwartz space on $\mathbb{R}^{d}$. The space $\mathcal{S}^{\prime}\left(\mathbb{R}^{d}\right)$ is the dual of $\mathcal{S}\left(\mathbb{R}^{d}\right)$, that is the space of tempered distributions. We want to work with a white noise measure, which is constructed on the nuclear algebra $\widetilde{\mathcal{S}}^{\prime}(X)$, introduced in [18]. The space $\widetilde{\mathcal{S}}(X)$ is defined as the quotient algebra

$$
\widetilde{\mathcal{S}}(X)=\mathcal{S}(X) / \mathcal{N}_{\pi},
$$

where $\mathcal{S}(X)$ is a subspace of $\mathcal{S}(X)$, given by

$$
\mathcal{S}(X):=\left\{\varphi \in \mathcal{S}\left(\mathbb{R}^{2}\right): \varphi(t, 0)=\left(\frac{\partial}{\partial x} \varphi\right)(t, 0)=0\right\}
$$

and where the closed ideal $\mathcal{N}_{\pi}$ in $\mathcal{S}(X)$ is defined as

$$
\mathcal{N}_{\pi}:=\left\{\phi \in \mathcal{S}(X):\|\phi\|_{L^{2}(\pi)}=0\right\} .
$$

The space $\widetilde{\mathcal{S}}(X)$ is a (countably Hilbertian) nuclear algebra. We indicate by $\widetilde{\mathcal{S}}^{\prime}(X)$ its dual.

From the Bochner-Minlos theorem we deduce that there exists a unique probability measure $\mu$ on the Borel sets of $\widetilde{\mathcal{S}}^{\prime}(X)$ such that

$$
\int_{\widetilde{\mathcal{S}}^{\prime}(X)} e^{i\langle\omega, \phi\rangle} d \mu(\omega)=\exp \left(\int_{X}\left(e^{i \phi}-1\right) d \pi\right)
$$

for all $\phi \in \widetilde{\mathcal{S}}(X)$, where $\langle\omega, \phi\rangle:=\omega(\phi)$ denotes the action of $\omega \in \widetilde{\mathcal{S}}^{\prime}(X)$ on $\phi \in \widetilde{\mathcal{S}}(X)$. The measure $\mu$ on $\Omega=\widetilde{\mathcal{S}}^{\prime}(X)$ is called the (pure jump) Lévy white noise probability measure. 
In the sequel we consider a compensated Poisson random measure

$$
\widetilde{N}(d t, d x)=N(d t, d x)-\nu(d x) d t
$$

associated with a Lévy process $L(t)$, which is defined on the white noise probability space

$$
(\Omega, \mathcal{F}, P)=\left(\widetilde{\mathcal{S}}^{\prime}(X), \mathcal{B}\left(\widetilde{\mathcal{S}}^{\prime}(X)\right), \mu\right)
$$

By using generalized Charlier polynomials $C_{n}(\omega) \in\left(\widetilde{\mathcal{S}}(X)^{\widehat{\otimes} n}\right)^{\prime}$ (dual of the $n$-th completed symmetric tensor product of $\widetilde{\mathcal{S}}(X)$ with itself) it is possible to construct an orthogonal $L^{2}(\mu)$-basis $\left\{\mathcal{K}_{\alpha}(\omega)\right\}_{\alpha \in \mathcal{J}}$ defined by

$$
\mathcal{K}_{\alpha}(\omega)=\left\langle C_{|\alpha|}(\omega), \delta^{\widehat{\otimes} \alpha}\right\rangle,
$$

where $\mathcal{J}$ is the multiindex set of all $\alpha=\left(\alpha_{1}, \alpha_{2}, \ldots\right)$ with finitely many non-zero components $\alpha_{i} \in \mathbb{N}_{0}$. The symbol $\delta^{\widehat{\otimes} \alpha}$ denotes the symmetrization of $\delta_{1}^{\otimes \alpha_{1}} \otimes \ldots \otimes \delta_{j}^{\otimes \alpha_{j}}$, where $\left\{\delta_{j}\right\}_{j \geq 1} \subset \widetilde{\mathcal{S}}(X)$ is an orthonormal basis of $L^{2}\left(\mathbb{R} \times \mathbb{R}_{0}, d t \nu(d x)\right)$.

So every $X \in L^{2}(\mu)$ has the unique representation

$$
X=\sum_{\alpha \in \mathcal{J}} c_{\alpha} \mathcal{K}_{\alpha}
$$

with Fourier coefficients $c_{\alpha} \in \mathbb{R}$. Moreover we have the isometry

$$
\|X\|_{L^{2}(\mu)}^{2}=\sum_{\alpha \in \mathcal{J}} \alpha ! c_{\alpha}^{2}
$$

with $\alpha !:=\alpha_{1} ! \alpha_{1} ! \ldots$ for $\alpha \in \mathcal{J}$. The Lévy-Hida test function space $(\mathcal{S})$ consists of all $f=\sum_{\alpha \in \mathcal{J}} c_{\alpha} \mathcal{K}_{\alpha} \in L^{2}(\mu)$ such that

$$
\|f\|_{0, k}^{2}:=\sum_{\gamma \in \mathcal{J}^{m}} \alpha ! c_{\alpha}^{2}(2 \mathbb{N})^{k \alpha}<\infty
$$

holds for all $k \in \mathbb{N}_{0}$ with weights $(2 \mathbb{N})^{k \alpha}=(2 \cdot 1)^{k \alpha_{1}}(2 \cdot 2)^{k \alpha_{2}} \ldots(2 \cdot l)^{k \alpha_{l}}$, if Index $(\alpha):=l$. The space $(\mathcal{S})$ is given the projective topology, induced by the norms $\left(\|\cdot\|_{0, k}\right)_{k \in \mathbb{N}_{0}}$ in (2.8). The Lévy-Hida distribution space, denoted by $(\mathcal{S})^{*}$ is the topological dual of $(\mathcal{S})$. So we obtain the following Gel'fand triple

$$
(\mathcal{S}) \hookrightarrow L^{2}(\mu) \hookrightarrow(\mathcal{S})^{*} .
$$

We can endow $(\mathcal{S})^{*}$ with the structure of a topological algebra by introducing the Wick product $\diamond$, defined by

$$
\left(\mathcal{K}_{\alpha} \diamond \mathcal{K}_{\beta}\right)(\omega)=\left(\mathcal{K}_{\alpha+\beta}\right)(\omega), \alpha, \beta \in \mathcal{J} .
$$

The product is linearly extensible to $(\mathcal{S})^{*} \times(\mathcal{S})^{*}$. It can be proven e.g. that

$$
\left\langle C_{n}(\omega), f_{n}\right\rangle \diamond\left\langle C_{m}(\omega), g_{m}\right\rangle=\left\langle C_{n+m}(\omega), f_{n} \widehat{\otimes} g_{m}\right\rangle
$$


for $f_{n} \in \widetilde{\mathcal{S}}(X)^{\widehat{\otimes} n}$ and $g_{m} \in \widetilde{\mathcal{S}}(X)^{\widehat{\otimes} m}$ (see [18]).

A nice feature of the Lévy-Hida distribution space is that it carries the white noise $\dot{\widetilde{N}}(t, x)$ of the Poisson random measure $\widetilde{N}(d t, d x)$, that is the formal Radon-Nikodym derivative of $\widetilde{N}(d t, d x)$ defined as

$$
\dot{\widetilde{N}}(t, x)=\sum_{k \geq 1} \delta_{k}(t, x) \mathcal{K}_{\alpha}(\omega)
$$

is in $(\mathcal{S})^{*} d t \nu(d x)$-a.e. The Wick product relates to stochastic integrals w.r.t. to $\widetilde{N}(d t, d x)$ in the following way: If $Y(t, x, \omega)$ is a predictable process, fulfilling the condition $E \int_{0}^{T} \int_{\mathbb{R}_{0}} Y^{2}(t, z, \omega) d t \nu(d z)<\infty$, then $Y(t, z, \omega) \diamond \dot{\widetilde{N}}(t, z)$ is $\lambda \times \nu$-Bochner integrable in $(\mathcal{S})^{*}$ and

$$
\int_{0}^{T} \int_{\mathbb{R}_{0}} Y(t, z, \omega) \tilde{N}(d t, d x)=\int_{0}^{T} \int_{\mathbb{R}_{0}} Y(t, z, \omega) \diamond \stackrel{\tilde{N}}{ }(t, z) d t \nu(d z) .
$$

An analogous relation is also valid for the Brownian motion. See [18] or [21] for definitions.

One of our main tools in the study of Lévy-Itô diffusions is the Lévy Hermite transform $\mathcal{H}$, which is used to give a characterization of distributions in $(\mathcal{S})^{*}$ (see characterization theorem 2.3.8 in [18]). Similar to the Gaussian case the definition of $\mathcal{H}$ rests on the basis $\left\{\mathcal{K}_{\alpha}(\omega)\right\}_{\alpha \in \mathcal{J}}$ in (2.6). The Lévy Hermite transform of $X(\omega)=$ $\sum_{\alpha} c_{\alpha} \mathcal{K}_{\alpha}(\omega) \in(\mathcal{S})^{*}$, denoted by $\mathcal{H} X$ or $\widetilde{X}$, is defined by

$$
\mathcal{H} X(z)=\tilde{X}(z)=\sum_{\alpha} c_{\alpha} z^{\alpha} \in \mathbb{C},
$$

where $z=\left(z_{1}, z_{2}, \ldots\right) \in \mathbb{C}^{\mathbb{N}}$, i.e. in the space of $\mathbb{C}$-valued sequences, and where $z^{\alpha}=z_{1}^{\alpha_{1}} z_{2}^{\alpha_{2}} \ldots$ We have that $\mathcal{H} X(z)$ in (2.13) is absolutely convergent on the infinite dimensional neighborhood

$$
\mathbb{K}_{q}(R):=\left\{\left(z_{1}, z_{2}, \ldots\right) \in \mathbb{C}^{\mathbb{N}}: \sum_{\alpha \neq 0}\left|z^{\alpha}\right|^{2}(2 \mathbb{N})^{q \alpha}<R^{2}\right\}
$$

for some $0<q \leq R<\infty$. For example, the Hermite transform of $\dot{\widetilde{N}}(t, x)$ can be evaluated as

$$
\mathcal{H}(\dot{\widetilde{N}}(t, x))(z)=\sum_{k \geq 1} \delta_{k}(t, x) z_{k} .
$$

The Hermite transform translates the Wick product into an ordinary (complex) product, that is

$$
\mathcal{H}(X \diamond Y)(z)=\mathcal{H}(X)(z) \cdot \mathcal{H}(Y)(z) .
$$

As a consequence of theorem 2.3.8 in [18] the last relation can be generalized to Wick versions of complex analytical functions $g$ : If the function $g: \mathbb{C} \longrightarrow \mathbb{C}$ can be expanded 
into a Taylor series around $\xi_{0}=\mathcal{H}(X)(0)$ with real valued coefficients, then there exists a unique distribution $Y \in(\mathcal{S})^{*}$ such that

$$
\mathcal{H}(Y)(z)=g(\mathcal{H}(X)(z))
$$

on $\mathbb{K}_{q}(R)$ for some $0<q \leq R<\infty$. We set $g^{\diamond}(X)=Y$.

For example, the Wick version of the exponential function exp can be written as

$$
\exp ^{\diamond} X=\sum_{n \geq 0} \frac{1}{n !} X^{\diamond n}
$$

Let us now outline how the preceding concepts and results can be generalized to capture the case of Lévy processes with Brownian motion and a pure jump part (see [23]). Indicate by $\mu_{G}$ the Gaussian white noise measure on the measurable space

$$
\left(\Omega_{G}, \mathcal{F}_{G}\right)=\left(\mathcal{S}^{\prime}(\mathbb{R}), \mathcal{B}\left(\mathcal{S}^{\prime}(\mathbb{R})\right)\right)
$$

Further recall the construction of the orthogonal $L^{2}\left(\mu_{G}\right)$ basis $\left\{H_{\alpha}(\omega)\right\}_{\alpha \in J}$, given by

$$
\mathcal{H}_{\alpha}(\omega)=\prod_{j \geq 1} h_{\alpha_{j}}\left(\left\langle\omega, \xi_{j}\right\rangle\right)
$$

where $\langle\omega, \cdot\rangle=\omega(\cdot)$ and where $\xi_{j}$ resp. $h_{j}, j=1,2, \ldots$ are the Hermite functions resp. Hermite polynomials. Using $\mu_{J}$ to denote the pure jump white noise measure on $\left(\Omega_{J}, \mathcal{F}_{J}\right)=\left(\widetilde{\mathcal{S}}^{\prime}(X), \mathcal{B}\left(\widetilde{\mathcal{S}}^{\prime}(X)\right)\right)$, we can define the Lévy white noise measure $\mu$ as the product measure $\mu_{G} \times \mu_{J}$ on

$$
(\Omega, \mathcal{F})=\left(\Omega_{G} \times \Omega_{J}, \mathcal{F}_{G} \otimes \mathcal{F}_{J}\right)
$$

Set

$$
\mathcal{L}_{\gamma}(\omega)=\mathcal{L}_{\gamma}\left(\omega_{1}, \omega_{2}\right)=\mathcal{H}_{\alpha}\left(\omega_{1}\right) \mathcal{K}_{\beta}\left(\omega_{2}\right)
$$

if $\gamma=(\alpha, \beta) \in \mathcal{I}:=\mathcal{J}^{2}$. Thus $\left(\mathcal{L}_{\gamma}(\omega)_{\gamma \in \mathcal{I}}\right.$ constitutes an $L^{2}(\mu)$-basis with norm expression

$$
\left\|\mathcal{L}_{\gamma}\right\|_{L^{2}(\mu)}^{2}=\gamma !
$$

where $\gamma !:=\alpha ! \beta$ ! for $\gamma=(\alpha, \beta) \in \mathcal{I}$.

As in the pure jump setting, we employ the basis $\left(\mathcal{L}_{\gamma}(\omega)_{\gamma \in \mathcal{I}}\right.$ to establish the concepts of Hida space, Wick product or Hermite transform to the mixture of Gaussian and pure jump Lévy noise. We also define the white noise $\dot{B}_{t}$ of Brownian motion as an element in the Hida distribution space:

$$
\dot{B}_{t}:=\sum_{k} \xi_{k}(t) H_{\epsilon_{k}} .
$$

Finally note that by choosing an appropriate basis the above described white noise theory can be established on any time interval $[0, T]$ instead of the complete time line $\mathbb{R}$ (which is used in the next section). 


\section{Explicit representation of strong solutions of Lévy-Itô diffusions}

Let $(\Omega, \mathcal{F}, \mu)$ be a white noise probability space as defined in 2.19 with time horizon $[0, T]$, corresponding Brownian motion $B_{t}$ and Lévy jump measure $N(d t, d x)$. We denote by $\left\{\mathcal{F}_{t}\right\}_{t>0}$ the augmented filtration generated by $B_{t}$ and $N(d t, d x)$. In the sequel of this paper we assume the Lévy measure $\nu(d x)$ corresponding to $N(d t, d x)$ to integrate $x^{2}$ and to be equivalent to the Lebesgue measure, that is

$$
\nu(d x)=\varphi(x) d x,
$$

where $\varphi(x)$ is strictly positive. Further, we require the corresponding Lévy process to be of finite variation, which means we have the following representation of the uncompensated Lévy process:

$$
L_{t}=\int_{0}^{t} \int_{\mathbb{R}_{0}} x N(d s, d x)
$$

We also presume that there exists an orthonormal basis $\left\{\delta_{k}\right\}_{k \in \mathbb{N}}$ of $L^{2}(\pi)$ such that

$$
\sup _{s, x}\left|\delta_{k}(s, x)\right|=O(1) \text { for } k \longrightarrow \infty .
$$

We then consider the following Lévy-Itô diffusion

$$
\begin{aligned}
d Y_{t} & =b\left(Y_{t-}\right) d t+\sigma\left(Y_{t-}\right) d B_{t}+\gamma\left(Y_{t-}\right) d L_{t} \\
& =b\left(Y_{t-}\right) d t+\sigma\left(Y_{t-}\right) d B_{t}+\int_{\mathbb{R}_{0}} \gamma\left(Y_{t-}\right) x N(d t, d x), \\
Y_{0} & =y, 0 \leq t \leq T,
\end{aligned}
$$

where $b, \sigma: \mathbb{R} \longrightarrow \mathbb{R}$ and $\gamma: \mathbb{R} \longrightarrow \mathbb{R}_{+}$are measurable functions.

In this Section we suppose there exists a square integrable strong solution $Y_{t} \in$ $L^{2}(\mu)$ of (3.3), that is $Y_{t}$ fulfils the stochastic differential equation (3.3) and is adapted to the filtration $\mathcal{F}_{t}$. This is for example the case under the usual Lipschitz and linear growth assumptions on the coefficients). The objective of this Section is then to employ the white noise concepts presented in Section 2 in order to give an explicit representation of $Y_{t}$. To this end we first consider the pure jump case of equation (3.3), i.e. $b, \sigma \equiv 0$, before we state the result for the mixed Lévy-Itô diffusion.

REMARK 3.1. We remark that even if due to notational convenience we only consider time homogeneous coefficients in (3.3) in this paper, the concepts and techniques presented below go through without difficulties for coefficients that depend additionally on $t$. Also we point out that all results except Subsection 5.2 are valid in all finite dimensions.

Notation 3.2. Throughout the remaining parts of the paper we will use the symbol ${ }^{\widehat{ }}$ to define a copy $(\widehat{\Omega}, \widehat{\mathcal{F}}, \widehat{\mu})$ of our initial white noise space as well as to denote the corresponding copied objects on this new stochastic basis. For example, $\widehat{L}_{t}=\int_{0}^{t} \int_{\mathbb{R}_{0}} x N(\widehat{\omega}, d s, d x)$ denotes a Lévy process that is a copy of $L_{t}$ on the auxiliary probability space $(\widehat{\Omega}, \widehat{\mathcal{F}}, \widehat{\mu})$.

3.1. Pure jump diffusion. Let $Y_{t} \in L^{2}(\mu)$ be a unique strong solution of the Lévy process driven diffusion

$$
\begin{aligned}
d Y_{t} & =\gamma\left(Y_{t-}\right) d L_{t} \\
& =\int_{\mathbb{R}_{0}} \gamma\left(Y_{t-}\right) x N(d t, d x), Y_{0}=y, 0 \leq t \leq T .
\end{aligned}
$$


Assume the Lévy measure is such that

$$
\int_{0}^{T} \int_{\mathbb{R}_{0}}\left(\frac{\varphi\left(\frac{x}{\gamma\left(L_{s-}\right)}\right)}{\varphi(x) \gamma\left(L_{s-}\right)}-1\right)^{2} \nu(d x) d s<\infty
$$

and define the martingale

$$
V_{t}(\omega)=\int_{0}^{t} \int_{\mathbb{R}_{0}}\left\{\frac{\varphi\left(\frac{x}{\gamma\left(L_{s-}\right)}\right)}{\varphi(x) \gamma\left(L_{s-}\right)}-1\right\} \tilde{N}(\omega, d s, d x) .
$$

Consider the Doleans-Dade exponential $\mathcal{E}\left(V_{t}\right)$ and suppose it is a martingale. Using the white noise concepts presented in Section 2, we are able to derive a general solution formula for $Y_{t}$ in (3.4).

THEOREM 3.3. Let $\rho$ be a Borel measurable function from $\mathbb{R}$ to $\mathbb{R}$ such that $\rho\left(Y_{t}\right) \in$ $L^{2}(\mu)$. Then, under the above specified assumptions, $\rho\left(Y_{t}(\omega)\right)$ is given by

$$
E_{\widehat{\mu}}^{y}\left[\rho\left(\widehat{L}_{t}\right) J_{T}^{\diamond}\right]
$$

where

$$
\begin{aligned}
& J_{T}^{\diamond}=\exp ^{\diamond} \int_{0}^{T} \int_{\mathbb{R}_{0}} \log ^{\diamond}\left(\frac{\left(1+\dot{\widetilde{N}}\left(\omega, s, \frac{x^{\prime}}{\gamma\left(\widehat{L}_{s-}\right)}\right)\right) \varphi\left(\frac{x^{\prime}}{\gamma\left(\widehat{L}_{s-}\right)}\right)}{\varphi\left(x^{\prime}\right) \gamma\left(\widehat{L}_{s-}\right)}\right) N\left(\widehat{\omega}, d s, d x^{\prime}\right) \\
& \diamond \exp ^{\diamond} \int_{0}^{T} \int_{\mathbb{R}_{0}}\left(1-\frac{\left(1+\stackrel{\tilde{N}}{ }\left(\omega, s, \frac{x^{\prime}}{\gamma\left(\widehat{L}_{s-}\right)}\right)\right) \varphi\left(\frac{x^{\prime}}{\gamma\left(\widehat{L}_{s-}\right)}\right)}{\varphi\left(x^{\prime}\right) \gamma\left(\widehat{L}_{s-}\right)}\right) \nu\left(d x^{\prime}\right) d s .
\end{aligned}
$$

Here the Wick product $\diamond$ is with respect to $\omega$ and the integrals occurring in (3.6) are (stochastic) Bochner integrals on the Lévy-Hida space.

Proof. The Hermite transform of the solution $Y_{t} \in L^{2}(\mu)$ can be expressed in the following way (see Theorem 2.7.10 in [9])

$$
\begin{aligned}
\mathcal{H}\left(\rho\left(Y_{t}\right)\right)(z) & =E_{\mu}^{y}\left[\rho\left(Y_{t}\right) \mathcal{E}\left(\int_{0}^{T} \int_{\mathbb{R}_{0}} \phi_{z}(s, x) \widetilde{N}(\omega, d s, d x)\right)\right] \\
& =E_{\widehat{\mu}}^{y}\left[\rho\left(\widehat{Y}_{t}\right) \mathcal{E}\left(\int_{0}^{T} \int_{\mathbb{R}_{0}} \phi_{z}(s, x) \widetilde{N}(\widehat{\omega}, d s, d x)\right)\right],
\end{aligned}
$$

where $\phi_{z}(s, x)=\mathcal{H}(\dot{\widetilde{N}}(s, x))(z)=\sum_{k} z_{k} \delta_{k}(s, x), \quad z \in \mathbb{C}_{c}^{\mathbb{N}}$ and $\tilde{N}(d s, d x)=N(d s, d x)-$ $\nu(d x) d s$. Note that

$$
\begin{aligned}
& \mathcal{E}\left(\int_{0}^{T} \int_{\mathbb{R}_{0}} \phi_{z}(s, x) \tilde{N}(d s, d x)\right) \\
& =\exp \left\{\int_{0}^{T} \int_{\mathbb{R}_{0}} \log \left(1+\phi_{z}(s, x)\right) N(d s, d x)-\int_{0}^{T} \int_{\mathbb{R}_{0}} \phi_{z}(s, x) \nu(d x) d s\right\} .
\end{aligned}
$$


The first step is to check that we can extract the Hermite transform in (3.7), that is that the Bochner integral

$$
\begin{aligned}
& E_{\widehat{\mu}}^{y}\left[\rho\left(\widehat{Y}_{t}\right) \exp ^{\diamond} \int_{0}^{T} \int_{\mathbb{R}_{0}} \log ^{\diamond}(1+\stackrel{\widetilde{N}}{ }(\omega, s, x)) N(\widehat{\omega}, d s, d x)\right.
\end{aligned}
$$

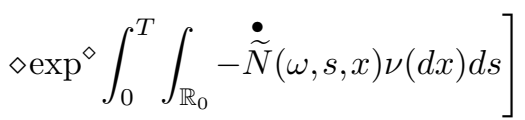

exists. For this purpose it is sufficient to show that

$$
E_{\widehat{\mu}}^{y}\left[\sup _{z \in \mathbb{K}_{q}(R)}\left|\rho\left(\widehat{Y}_{t}\right) \mathcal{E}\left(\int_{0}^{T} \int_{\mathbb{R}_{0}} \phi_{z}(s, x) \tilde{N}(\widehat{\omega}, d s, d x)\right)\right|\right]<\infty
$$

for some $q, R$. We find the estimate

$$
\sup _{z \in \mathbb{K}_{q}(R)}\left|\phi_{z}(s, x)\right| \leq R\|\stackrel{\dot{\widetilde{N}}(s, x)}{ }\|_{0,-2} d s \times \nu-\text { a.e. }
$$

where $\|\cdot\|_{0,-2}$ is the norm for distributions, which corresponds to the norm $\|\cdot\|_{0,2}$ (see Section 2). Then by (3.2) there exists an $M>0$ such that

$$
\sup _{z \in \mathbb{K}_{q}(R)}\left|\phi_{z}(s, x)\right| \leq R M d s \times \nu-\text { a.e. }
$$

So we get

$$
\begin{aligned}
& E_{\widehat{\mu}}^{y}\left[\sup _{z \in \mathbb{K}_{q}(R)}\left|\rho\left(\widehat{Y}_{t}\right) \mathcal{E}\left(\int_{0}^{T} \int_{\mathbb{R}_{0}} \phi_{z}(s, x) \tilde{N}(\widehat{\omega}, d s, d x)\right)\right|\right] \\
& \leq E_{\widehat{\mu}}^{y}\left[\left|\rho\left(\widehat{Y}_{t}\right)\right| \mathcal{E}\left(\int_{0}^{T} \int_{\mathbb{R}_{0}} R\|\dot{\widetilde{N}}(s, x)\|_{0,-2} \tilde{N}(\widehat{\omega}, d s, d x)\right)\right. \\
& \left.\exp \int_{0}^{T} \int_{\mathbb{R}_{0}} 2 R\|\stackrel{\ddot{N}}{N}(s, x)\|_{0,-2} \nu(d x) d s\right] .
\end{aligned}
$$

Since

$$
\int_{0}^{T} \int_{\mathbb{R}_{0}} 2 R\|\stackrel{\dot{\widetilde{N}}}{ }(s, x)\|_{0,-2}^{2} \nu(d x) d s<\infty
$$

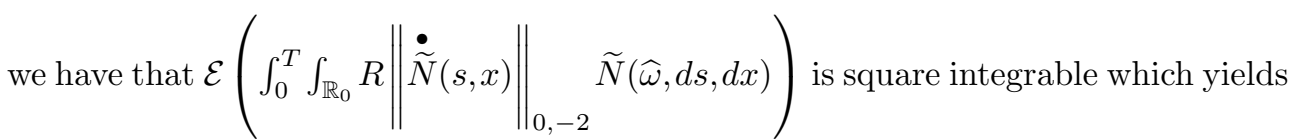
with the help of Hölder

$$
\begin{aligned}
& E_{\widehat{\mu}}^{y}\left[\sup _{z \in \mathbb{K}_{q}(R)}\left|\rho\left(\widehat{Y}_{t}\right) \mathcal{E}\left(\int_{0}^{T} \int_{\mathbb{R}_{0}} \phi_{z}(s, x) \tilde{N}(\widehat{\omega}, d s, d x)\right)\right|\right] \\
& \leq \text {.const } \cdot E_{\widehat{\mu}}^{y}\left[\rho\left(\widehat{Y}_{t}\right)^{2}\right] E_{\widehat{\mu}}^{y}\left[\mathcal{E}\left(\int_{0}^{T} \int_{\mathbb{R}_{0}} R\|\stackrel{\tilde{N}}{N}(s, x)\|_{0,-2} \tilde{N}(\widehat{\omega}, d s, d x)\right)^{2}\right]<\infty
\end{aligned}
$$


and (3.8) is well defined.

Next, in virtue of the Girsanov theorem for random measures (see [10]), it follows that we can rewrite $(3.7)$ as

$$
\mathcal{H}\left(\rho\left(Y_{t}\right)\right)(z)=E_{Q}^{y}\left[\rho\left(\widehat{Y}_{t}\right)\right]
$$

where $Q$ is the probability measure, given by

$$
d Q=\mathcal{E}\left(\int_{0}^{T} \int_{\mathbb{R}_{0}} \phi_{z}(s, x) \tilde{N}(d s, d x)\right) d \widehat{\mu}
$$

and where $\widehat{Y}$ under $Q$ is a jump process whose jump measure has the predictable compensation

$$
\nu^{*}(d s, d x)=\left(1+\phi_{z}(s, x)\right) \nu(d x) d s .
$$

The process $Y_{t}$ can be rewritten in terms of its jump measure denoted by $N^{\prime}(d t, d x)$ :

$$
d Y_{t}=\int_{\mathbb{R}_{0}} x N^{\prime}(d t, d x)
$$

where the $Q$-compensation $\nu^{\prime}(\omega, d s, d x)$ of $N^{\prime}(d s, d x)$ is given by the relation

$$
\int_{0}^{T} \int_{\mathbb{R}_{0}} f(x) \nu^{\prime}(\omega, d s, d x)=\int_{0}^{T} \int_{\mathbb{R}_{0}} f\left(\gamma\left(Y_{s-}\right) x\right)\left(1+\phi_{z}(s, x)\right) \nu(d x) d s
$$

for all $\nu^{\prime}$-integrable $f$. The substitution $x^{\prime}=\gamma\left(Y_{s-}\right) x$ on the right hand side of the latter relation yields

$$
\int_{0}^{T} \int_{\mathbb{R}_{0}} f(x) \nu^{\prime}(\omega, d s, d x)=\int_{0}^{T} \int_{\mathbb{R}_{0}} f\left(x^{\prime}\right) \pi_{z}\left(\omega, s, x^{\prime}\right) d x^{\prime} d s
$$

where

$$
\pi_{z}\left(\omega, s, x^{\prime}\right)=\left(1+\phi_{z}\left(s, \frac{x^{\prime}}{\gamma\left(Y_{s-}\right)}\right)\right) \cdot \varphi\left(\frac{x^{\prime}}{\gamma\left(Y_{s-}\right)}\right) \cdot \frac{1}{\gamma\left(Y_{s-}\right)}
$$

Note that with

$$
\theta_{z}\left(\widehat{\omega}, s, x^{\prime}\right)=\frac{\varphi\left(x^{\prime}\right)}{\pi_{z}\left(\widehat{\omega}, s, x^{\prime}\right)}-1
$$

we have that

$$
E_{Q}^{y}\left[\mathcal{E}\left(\int_{0}^{t} \int_{\mathbb{R}_{0}} \theta_{z}\left(\widehat{\omega}, s, x^{\prime}\right)\left(N^{\prime}-\nu^{\prime}\right)\left(\widehat{\omega}, d s, d x^{\prime}\right)\right)\right]=1
$$

because we assumed $\mathcal{E}\left(M_{t}\right)$ to be a martingale. By invoking the Girsanov theorem 
again, we get that

$$
\begin{aligned}
& E_{Q}^{y}\left[\rho\left(\widehat{Y}_{t}\right)\right] \\
& =E_{Q}^{y}\left[\widehat{Y}_{t} \mathcal{E}\left(\int_{0}^{T} \int_{\mathbb{R}_{0}} \theta_{z}\left(\widehat{\omega}, s, x^{\prime}\right)\left(N^{\prime}-\nu^{\prime}\right)\left(\widehat{\omega}, d s, d x^{\prime}\right)\right)\right. \\
& \left.\mathcal{E}^{-1}\left(\int_{0}^{T} \int_{\mathbb{R}_{0}} \theta_{z}\left(\widehat{\omega}, s, x^{\prime}\right)\left(N^{\prime}-\nu^{\prime}\right)\left(\widehat{\omega}, d s, d x^{\prime}\right)\right)\right] \\
& =E_{Q^{\prime}}^{y}\left[\widehat{Y}_{t} \mathcal{E}^{-1}\left(\int_{0}^{T} \int_{\mathbb{R}_{0}} \theta_{z}\left(\widehat{\omega}, s, x^{\prime}\right)\left(N^{\prime}-\nu^{\prime}\right)\left(\widehat{\omega}, d s, d x^{\prime}\right)\right)\right] \\
& =E_{Q^{\prime}}^{y}\left[\rho\left(\widehat{Y}_{t}\right) \exp \left\{\int_{0}^{T} \int_{\mathbb{R}_{0}} \log \left(\frac{1}{\theta_{z}\left(\widehat{\omega}, s, x^{\prime}\right)+1} N^{\prime}\left(\widehat{\omega}, d s, d x^{\prime}\right)\right)\right\}\right. \\
& \left.\cdot \exp \left\{\int_{0}^{T} \int_{\mathbb{R}_{0}}\left(1-\frac{1}{\theta_{z}\left(\widehat{\omega}, s, x^{\prime}\right)+1}\right) \nu\left(d x^{\prime}\right) d s\right\}\right] \\
& =E_{\widehat{\mu}}^{y}\left[\rho\left(\widehat{L}_{t}\right) \exp \left\{\int_{0}^{T} \int_{\mathbb{R}_{0}} \log \left(\frac{\left(1+\phi\left(s, \frac{x^{\prime}}{\gamma\left(\widehat{L}_{s-}\right)}, z\right)\right) \varphi\left(\frac{x^{\prime}}{\gamma\left(\widehat{L}_{s-}\right)}\right)}{\varphi\left(x^{\prime}\right) \gamma\left(\widehat{L}_{s-}\right)}\right) N\left(\widehat{\omega}, d s, d x^{\prime}\right)\right\}\right. \\
& \left.\cdot \exp \left\{\int_{0}^{T} \int_{\mathbb{R}_{0}}\left(1-\frac{\left(1+\phi_{z}\left(s, \frac{x^{\prime}}{\gamma\left(\widehat{L}_{s-}\right)}\right)\right) \varphi\left(\frac{x^{\prime}}{\gamma\left(\widehat{L}_{s-}\right)}\right)}{\varphi\left(x^{\prime}\right) \gamma\left(\widehat{L}_{s-}\right)}\right) \nu\left(d x^{\prime}\right) d s\right\}\right]
\end{aligned}
$$

where

$$
d Q^{\prime}=\mathcal{E}\left(\int_{0}^{T} \int_{\mathbb{R}_{0}} \theta_{z}\left(\widehat{\omega}, s, x^{\prime}\right)\left(N^{\prime}-\nu^{\prime}\right)\left(\widehat{\omega}, d s, d x^{\prime}\right)\right) d Q
$$

Here we have employed the relation (3.9) to derive identity (3.10), and identity (3.11) is due to the fact that $Y_{t}$ under $Q^{\prime}$ has the same law as $L_{t}$ under $\mu$.

Therefore we obtain the relation

$$
\begin{array}{r}
\mathcal{H}\left(\rho\left(Y_{t}\right)\right)(z)= \\
=E_{\widehat{\mu}}^{y}\left[\rho\left(\widehat{L}_{t}\right) \exp \left\{\int_{0}^{T} \int_{\mathbb{R}_{0}} \log \left(\frac{\left(1+\phi_{z}\left(s, \frac{x^{\prime}}{\gamma\left(\hat{L}_{s-}\right)}\right) \varphi\left(\frac{x^{\prime}}{\gamma\left(\widehat{L}_{s-}\right)}\right)\right.}{\varphi\left(x^{\prime}\right) \gamma\left(\widehat{L}_{s-}\right)}\right) N\left(\widehat{\omega}, d s, d x^{\prime}\right)\right\}\right. \\
\left.\cdot \exp \left\{\int_{0}^{T} \int_{\mathbb{R}_{0}}\left(1-\frac{\left(1+\phi_{z}\left(s, \frac{x^{\prime}}{\gamma\left(\widehat{L}_{s-}\right)}\right) \varphi\left(\frac{x^{\prime}}{\gamma\left(\widehat{L}_{s-}\right)}\right)\right.}{\varphi\left(x^{\prime}\right) \gamma\left(\widehat{L}_{s-}\right)}\right) \nu\left(d x^{\prime}\right) d s\right\}\right]
\end{array}
$$

and also 


$$
\begin{array}{r}
E_{\widehat{\mu}}^{y} \sup _{z \in \mathbb{K}_{q}(R)}\left|\rho\left(\widehat{L}_{t}\right) \exp \left\{\int_{0}^{T} \int_{\mathbb{R}_{0}} \log \left(\frac{\left(1+\phi_{z}\left(s, \frac{x^{\prime}}{\gamma\left(\widehat{L}_{s-}\right)}\right)\right) \varphi\left(\frac{x^{\prime}}{\gamma\left(\widehat{L}_{s-}\right)}\right)}{\varphi\left(x^{\prime}\right) \gamma\left(\widehat{L}_{s-}\right)}\right) N\left(\widehat{\omega}, d s, d x^{\prime}\right)\right\}\right| \\
\left.\cdot\left|\exp \left\{\int_{0}^{T} \int_{\mathbb{R}_{0}}\left(1-\frac{\left(1+\phi_{z}\left(s, \frac{x^{\prime}}{\gamma\left(\hat{L}_{s-}\right)}\right) \varphi\left(\frac{x^{\prime}}{\gamma\left(\widehat{L}_{s-}\right)}\right)\right.}{\varphi\left(x^{\prime}\right) \gamma\left(\widehat{L}_{s-}\right)}\right) \nu\left(d x^{\prime}\right) d s\right\}\right|\right] \\
=E_{\widehat{\mu}}^{y}\left[\sup _{z \in \mathbb{K}_{q}(R)}\left|\rho\left(\widehat{Y}_{t}\right) \mathcal{E}\left(\int_{0}^{T} \int_{\mathbb{R}_{0}} \phi_{z}(s, x) \widetilde{N}(\widehat{\omega}, d s, d x)\right)\right|\right]<\infty
\end{array}
$$

for $z \in \mathbb{K}_{q}(R)$ with some $0<q, R<\infty$. Consequently we can extract the Hermite transform on both sides of (3.12), which concludes the proof.

REMARK 3.4. A sufficient condition for (3.5) to hold is that $\nu(d x)$ has a singularity of order 1 around 0, i.e. $\varphi(x)=O(1 / x)$ when $x \rightarrow 0$. An important example of a Lévy process, which satisfies the above assumptions, is a class of Gamma processes. In this case one can e.g. choose $\left\{\xi_{n} \otimes \psi_{m}\right\}_{n, m \in \mathbb{N}}$ as an orthonormal basis of $L^{2}(\pi)$, where $\xi_{n}$ are Hermite functions and $\psi_{m}$ Laguerre functions of order $\frac{1}{2}$. Since it is known that $\xi_{n}, \psi_{m}$ are uniformly bounded [29] one sees that (3.2) is fulfilled.

REMARK 3.5. A set of sufficient conditions to ensure that $\mathcal{E}\left(V_{t}\right)$ is a martingale is that $\gamma$ is bounded and that

$$
M \geq \frac{\varphi\left(\frac{x}{\gamma(y)}\right)}{\varphi(x) \gamma(y)}>0
$$

holds for all $x, y \in \mathbb{R}$, where $M$ is a constant.

REMARK 3.6. The formula (3.3) necessitates stochastic integration of the form

$$
\int_{0}^{t} \int_{\mathbb{R}_{0}} \Phi(\omega, s, x) N(\omega, d s, d x)
$$

where $\Phi$ is a process on the conuclear space $(\mathcal{S})^{*}$ and $N$ is the Poisson random measure. For general information about stochastic integration on conuclear spaces with respect to Poisson random measures we refer to [12].

In particular, if $\rho=i d$ and $\mathcal{E}\left(M_{t}\right)$ is a martingale we get:

Corollary 3.7. The solution $Y_{t} \in L^{2}(\mu)$ in (3.1) has the form

$$
\begin{aligned}
& Y=E_{\widehat{\mu}}^{y}\left[\widehat { L } _ { t } \operatorname { e x p } ^ { \diamond } \left\{\int_{0}^{T} \int_{\mathbb{R}_{0}} \log ^{\diamond}\left(\frac{\left(1+\dot{\widetilde{N}}\left(\omega, s, \frac{x^{\prime}}{\gamma\left(\widehat{L}_{s-1}\right)}\right)\right) \varphi\left(\frac{x^{\prime}}{\gamma\left(\widehat{L}_{s-}\right)}\right)}{\varphi\left(x^{\prime}\right) \gamma\left(\widehat{L}_{s-}\right)}\right) N\left(\widehat{\omega}, d s, d x^{\prime}\right)\right.\right. \\
& \diamond \exp ^{\diamond}\left\{\int_{0}^{T} \int_{\mathbb{R}_{0}}\left(1-\frac{\left(1+\dot{\widetilde{N}}\left(\omega, s, \frac{x^{\prime}}{\gamma\left(\widehat{L}_{s-}\right.}\right)\right) \varphi\left(\frac{x^{\prime}}{\gamma\left(\widehat{L}_{s-}\right)}\right)}{\varphi\left(x^{\prime}\right) \gamma\left(\widehat{L}_{s-}\right)}\right) \nu\left(d x^{\prime}\right) d s\right] .
\end{aligned}
$$


3.2. Mixed Lévy-Itô diffusion. Let now $Y_{t} \in L^{2}(\mu)$ be a unique strong solution of the Lévy-Itô diffusion (3.3) where our stochastic basis is the white noise space (2.19). In addition to the above specified assumptions on coefficients and the Lévy measure we restrict $\sigma$ to be a strictly positive and continuously differentiable, and $\nu(d x)$ to have only support on the positive half line. Further we consider the strictly increasing function $\Lambda: \mathbb{R} \rightarrow \mathbb{R}$, given by

$$
\Lambda(y):= \begin{cases}\int_{x}^{y} \frac{1}{\sigma(u)} d u, & y>x \\ -\int_{y}^{x} \frac{1}{\sigma(u)} d u, & y \leq x,\end{cases}
$$

and define the function $\gamma^{*}(y, x)$ as

$$
\gamma^{*}(y, x)=\Lambda\left(\Lambda^{-1}(y)+\gamma\left(\Lambda^{-1}(y)\right) x\right)-y,
$$

which is invertible in $x>0$ for all $y$. Assume

$$
U_{t}(\omega)=\int_{0}^{t}\left(\left(\frac{b}{\sigma}\right)\left(\Lambda^{-1}\left(B_{s}\right)\right)-\frac{1}{2} \sigma^{\prime}\left(\Lambda^{-1}\left(B_{s}\right)\right)\right) d B_{s}
$$

and

$$
V_{t}(\omega)=\int_{0}^{t} \int_{\mathbb{R}_{0}}\left(\frac{\varphi\left(\gamma^{*-1}\left(\widehat{L}_{s-}, x^{\prime}\right)\right)}{\varphi\left(x^{\prime}\right)} \frac{\partial \gamma^{*-1}}{\partial x^{\prime}}\left(\widehat{L}_{s-}, x^{\prime}\right)\right) \widetilde{N}(\omega, d s, d x)
$$

as well as the corresponding Doleans-Dade exponentials $\mathcal{E}\left(U_{t}\right)$ and $\mathcal{E}\left(V_{t}\right)$ are martingales. Then we can state the analogue to Theorem 3.3:

TheOREM 3.8. Let $\rho$ be a Borel measurable function from $\mathbb{R}$ to $\mathbb{R}$ such that $\rho\left(Y_{t}\right) \in$ $L^{2}(\mu)$. Then, under the above specified assumptions, $\rho\left(Y_{t}(\omega)\right)$ is given by

$$
E_{\widehat{\mu}}^{y}\left[\rho\left(\Lambda^{-1}\left(\widehat{B}_{t}+\widehat{L}_{t}\right)\right) M_{T}^{\diamond} \diamond J_{T}^{\diamond}\right]
$$

where

$$
\begin{aligned}
M_{T}^{\diamond}=\exp ^{\diamond} & \left\{\int_{0}^{T}\left(\dot{B}_{s}(\omega)+\left(\frac{b}{\sigma}\right)\left(\Lambda^{-1}\left(\widehat{B}_{s}\right)\right)-\frac{1}{2} \sigma^{\prime}\left(\Lambda^{-1}\left(\widehat{B}_{s}\right)\right)\right) d \widehat{B}_{s}-\right. \\
& \left.\left.\frac{1}{2} \int_{0}^{T}\left(\dot{B}_{s}(\omega)+\left(\frac{b}{\sigma}\right)\left(\Lambda^{-1}\left(\widehat{B}_{s}\right)\right)-\frac{1}{2} \sigma^{\prime}\left(\Lambda^{-1}\left(\widehat{B}_{s}\right)\right)\right)\right)^{\diamond 2} d s\right\}
\end{aligned}
$$

and

$$
\begin{array}{r}
J_{T}^{\diamond}=\exp ^{\diamond}\left\{\int _ { 0 } ^ { T } \int _ { \mathbb { R } _ { 0 } } \operatorname { l o g } ^ { \diamond } \left(\left(1+\dot{\widetilde{N}}\left(\omega, s, \gamma^{*-1}\left(\widehat{L}_{s-}, x^{\prime}\right)\right)\right)\right.\right. \\
\left.\left.\left.\frac{\varphi\left(\gamma^{*-1}\left(\widehat{L}_{s-}, x^{\prime}\right)\right)}{\varphi\left(x^{\prime}\right)} \frac{\partial \gamma^{*-1}}{\partial x^{\prime}}\left(\widehat{L}_{s-}, x^{\prime}\right)\right)\right) N\left(\widehat{\omega}, d s, d x^{\prime}\right)\right\} \\
\diamond \exp ^{\diamond}\left\{\int _ { 0 } ^ { T } \int _ { \mathbb { R } _ { 0 } } \left(1-\left(1+\dot{\widetilde{N}}\left(\omega, s, \gamma^{*-1}\left(\widehat{L}_{s-}, x^{\prime}\right)\right)\right)\right.\right. \\
\left.\left.\left.\frac{\varphi\left(\gamma^{*-1}\left(\widehat{L}_{s-}, x^{\prime}\right)\right)}{\varphi\left(x^{\prime}\right)} \frac{\partial \gamma^{*-1}}{\partial x^{\prime}}\left(\widehat{L}_{s-}, x^{\prime}\right)\right) \nu\left(d x^{\prime}\right) d s\right)\right\} .
\end{array}
$$


Also here the Wick product $\diamond$ is with respect to $\omega$ and the integrals occurring in (3.13) are (stochastic) Bochner integrals on the Lévy-Hida space.

Proof. W.l.o.g. we set $\rho=i d$. For $Z_{t}:=\Lambda\left(Y_{t}\right)$ Itô's Lemma implies that

$$
\begin{aligned}
Z_{t}= & \left(\left(\frac{b}{\sigma}\right)\left(\Lambda^{-1}\left(Z_{t}\right)\right)-\frac{1}{2} \sigma^{\prime}\left(\Lambda^{-1}\left(Z_{t}\right)\right)\right) d t+d B_{t}+ \\
& \int_{\mathbb{R}_{0}}\left(\Lambda\left(\Lambda^{-1}\left(Z_{t^{-}}\right)+\gamma\left(\Lambda^{-1}\left(Z_{t^{-}}\right)\right) x\right)-\Lambda\left(\Lambda^{-1}\left(Z_{t^{-}}\right)\right)\right) N(d t, d x) \\
= & b^{*}\left(Z_{t}\right) d t+d B_{t}+\int_{\mathbb{R}_{0}} \gamma^{*}\left(Z_{t^{-}}, x\right) N(d t, d x)
\end{aligned}
$$

where

$$
b^{*}(y)=\left(\frac{b}{\sigma}\right)\left(\Lambda^{-1}(y)\right)-\frac{1}{2} \sigma^{\prime}\left(\Lambda^{-1}(y)\right)
$$

and

$$
\gamma^{*}(y, x)=\Lambda\left(\Lambda^{-1}(y)+\gamma\left(\Lambda^{-1}(y)\right) x\right)-y .
$$

Observe that $\gamma^{*}(y, x) \neq 0$ for all $x, y$ and that for $y$ given $\gamma^{*}(y, x)$ is invertible in $x>0$.

To complete the proof it is sufficient to give the explicit representation for $Z_{t}$. Taking the Hermite transform we get

$$
\begin{aligned}
& \mathcal{H}\left(Z_{t}\right)(z) \\
= & E_{\widehat{\mu}}^{y}\left[\hat{Z}_{t} \mathcal{E}\left(\int_{0}^{T} \phi_{z}^{\prime}(t) d \widehat{B}_{t}\right) \cdot \mathcal{E}\left(\int_{0}^{T} \int_{\mathbb{R}_{0}} \phi_{z}(t, x) \widetilde{N}(\widehat{\omega}, d t, d x)\right)\right]
\end{aligned}
$$

where $\phi_{z}^{\prime}(s)=\mathcal{H}\left(\dot{B}_{s}\right)(z)=\sum_{k} z_{k} \xi_{k}(s)$ and $\phi_{z}(s, x)=\mathcal{H}(\stackrel{\tilde{N}}{(s, x)})(z)=\sum_{k} z_{k} \delta_{k}(s, x), z \in$ $\mathbb{C}_{c}^{\mathbb{N}}$.

The rest of the proof is analogous to the line of reasoning of Theorem 3.3.

4. Application I: Chaos expansions of diffusions

In [30]-[32] the author derives chaos expansions of SDE's of the type

$$
\begin{aligned}
d Y_{t} & =b\left(Y_{t}\right) d t+\sigma\left(Y_{t}\right) d B_{t} \\
Y_{0} & =y, 0 \leq t \leq T,
\end{aligned}
$$

where the kernels are determined by iteratively solving deterministic Cauchy problems. Of course there still remains the task to solve the Cauchy problems in order to calculate the chaos expansion. Further, this representation is only possible for diffusions whose coefficients fulfill certain smoothness conditions. In this Section we demonstrate how the formula (3.13) can be used to derive an explicit chaos expansion of SDE's of type (4.1) as soon as we have a strong solution $Y_{t} \in L^{2}(\mu)$ as in the previous Section.

Proposition 4.1. Under the assumptions specified in Theorem 3.8, $Y_{t}$ can be represented as

$$
Y_{t}=\sum_{\gamma \in \mathcal{J}} c_{\gamma}(t) \mathcal{H}_{\gamma}
$$


with explicit Fourier coefficients

$$
\begin{aligned}
& c_{\gamma}(t) \\
= & E_{\widehat{\mu}}^{y}\left[\Lambda^{-1}\left(\widehat{B}_{t}\right) \mathcal{E}\left(\int_{0}^{T} \vartheta_{s}(\widehat{\omega}) d \widehat{B}_{s}\right) \sum_{\alpha+\beta=\gamma} \frac{1}{\alpha ! \beta !}(-1)^{|\beta|}(\vartheta .(\widehat{\omega}), \eta)^{\beta} \mathcal{H}_{\alpha}(\widehat{\omega})\right],
\end{aligned}
$$

where

$$
\vartheta_{s}(\widehat{\omega}):=\frac{b\left(\Lambda^{-1}\left(\widehat{B}_{s}\right)\right)}{\sigma\left(\Lambda^{-1}\left(\widehat{B}_{s}\right)\right)}-\frac{1}{2} \sigma^{\prime}\left(\Lambda^{-1}\left(\widehat{B}_{s}\right)\right)
$$

and where

$$
(\vartheta \cdot(\widehat{\omega}), \eta)^{\beta}=\prod_{i \geq 1}\left(\vartheta \cdot(\widehat{\omega}), \eta_{i}\right)_{L^{2}}^{\beta_{i}}
$$

Proof. By using the Hermite transform it can be checked that

$$
\begin{aligned}
& \exp ^{\diamond}\left\{\int_{0}^{T} \dot{B}_{s}(\omega) d \widehat{B}_{s}(\widehat{\omega})-\frac{1}{2} \int_{0}^{T}\left(\dot{B}_{s}(\omega)\right)^{\diamond 2} d s\right\} \\
& =\sum_{\alpha} \frac{1}{\alpha !} \mathcal{H}_{\alpha}(\widehat{\omega}) \mathcal{H}_{\alpha}(\omega) .
\end{aligned}
$$

In addition we find

$$
\begin{aligned}
\exp ^{\diamond}\left\{\int_{0}^{T} \vartheta_{s}(\widehat{\omega}) \dot{B}_{s}(\omega) d s\right\} & =\exp ^{\diamond}\left\{\int_{0}^{T} \vartheta_{s}(\widehat{\omega}) d B_{s}(\omega)\right\} \\
& =\sum_{\beta} \frac{1}{\beta !}(\vartheta \cdot(\widehat{\omega}), \eta)^{\beta} \mathcal{H}_{\beta}(\omega) .
\end{aligned}
$$

Thus the statement of the theorem follows from (3.13).

Note that the Fourier coefficients are explicitly given since they are expressed in terms of Brownian motion. The analogue expansion of the jump diffusion (3.4) can not be given by Fourier coefficients expressed as expectations of only the Lévy process as an underlying process. This is due to the fact that a Lévy process under a measure change of Girsanov type in general can not be compensated to be again a Lévy process (as is the case for Brownian motion).

5. Application II: Existence of strong solutions of SDE's with irregular coefficients

In this Section we want to use the explicit representation of Lévy-Itô diffusions in order to examine questions around existence and uniqueness of strong solutions of SDE's

$$
\begin{aligned}
d Y_{t} & =b\left(Y_{t}\right) d t+\sigma\left(Y_{t}\right) d B_{t}, \\
Y_{0} & =y, 0 \leq t \leq T,
\end{aligned}
$$

where the drift coefficient $b$ is admitted to be irregular, i.e. we do not impose higher regularity like Lipschitzianity or Sobolev differentiability. Putting assumptions on 
$\sigma(y)$ as specified in Subsection 3.2, we have seen that we can w.l.o.g. assume $\sigma \equiv 1$, i.e. we will only consider distorted Brownian motions in the following. For the discontinuous case

$$
\begin{aligned}
d Y_{t-} & =b\left(Y_{t-}\right) d t+d B_{t}+\int_{\mathbb{R}_{0}} \gamma\left(Y_{t-}\right) d L_{t}, \\
Y_{0} & =y, 0 \leq t \leq T
\end{aligned}
$$

the corresponding results can be obtained under more restrictive conditions which we will point out informally as we go along.

In Subsection 5.1 we characterize the existence and uniqueness of strong solutions of (5.1) (resp. (5.2)) in terms of strong solutions corresponding to converging coefficients. In Subsection 5.2 we then apply this characterization in order to establish sufficient conditions for the existence and uniqueness of strong solutions. Actually, Subsection 5.2. is the only part in this paper that can not be generalized to $n$ dimensions because we rely on the formulation of a generalized comparison result of SDE's with irregular coefficients.

REMARK 5.1. It is important to note that the results of this paper do not depend on the choice of the white noise space as underlying probability space. The reason is that for any stochastic basis $\left(\Omega, P, \mathcal{F},\left\{\mathcal{F}_{t}\right\}_{0 \leq t \leq T}, B, L\right)$, where $\mathcal{F}_{t}$ is a complete filtration and $B$, resp. $L$, are an $\mathcal{F}_{t}$-Brownian motion, resp. $\mathcal{F}_{t}$-jump Lévy process, one can construct the corresponding concepts of stochastic distributions, white noise and Wick product of functionals. Consequently, our statements about strong solutions are true on any stochastic basis and we talk indeed about strong solutions in the usual sense of the definition.

In the light of remark 5.1 we present the following three facts of independent interest. Let $\left(\Omega, P, \mathcal{F},\left\{\mathcal{F}_{t}\right\}_{0 \leq t \leq T}, B, L\right)$ be a stochastic basis as in Remark 5.1 and denote by $\left\{\mathcal{G}_{t}\right\}_{0 \leq t \leq T}$ the completed filtration generated by $B$ and $L$.

Lemma 5.2. We can express the conditional expectation of $Y \in L^{2}(P)$ w.r.t $\mathcal{G}_{t}$ with the help of the techniques presented in Section 3:

$$
E_{\hat{P}}\left[\hat{Y} \bar{J}_{t}^{\diamond} \bar{M}_{t}^{\diamond}\right]=E_{\hat{P}}\left[E_{\hat{P}}\left[\hat{Y} \mid \hat{\mathcal{G}}_{t}\right] \bar{J}_{t}^{\diamond} \bar{M}_{t}^{\diamond}\right]=E_{P}\left[Y \mid \mathcal{G}_{t}\right]
$$

where

$$
\begin{gathered}
\bar{J}_{t}^{\diamond}=\exp ^{\diamond} \int_{0}^{t} \int_{\mathbb{R}_{0}} \log ^{\diamond}(1+\stackrel{\widetilde{N}}{ }(\omega, s, x)) N(\widehat{\omega}, d s, d x)-\int_{0}^{t} \int_{\mathbb{R}_{0}} \stackrel{\dot{\widetilde{N}}}{ }(\omega, s, x) \nu(d x) d s, \\
\bar{M}_{t}^{\diamond}=\exp ^{\diamond}\left(\int_{0}^{t} \dot{B}_{u}(\omega) d \widehat{B}_{u}-\frac{1}{2} \int_{0}^{t}\left(\dot{B}_{u}(\omega)\right)^{\diamond 2} d u\right) .
\end{gathered}
$$

LEMma 5.3. For a functional $Y=f(B, L) \in L^{2}(P)$ we have the following transformation property which will be crucial in our proofs

$$
\varphi(Y)=E_{\hat{P}}\left[\varphi(\hat{Y}) \bar{J}_{t}^{\diamond} \bar{M}_{t}^{\diamond}\right]
$$

for all measurable $\varphi: \mathbb{R} \rightarrow \mathbb{R}$ such that $\varphi(Y) \in L^{2}(P)$. 
These first two properties are seen immediately by taking the Hermite transform.

LEMmA 5.4. If $\left(\Omega^{\prime}, P^{\prime}, \mathcal{F}^{\prime},\left\{\mathcal{F}_{t}^{\prime}\right\}_{0 \leq t \leq T}, B^{\prime}, L^{\prime}\right)$ is another stochastic basis, then we can 'lift' a functional $Y^{\prime}=f\left(B^{\prime}, L^{\prime}\right) \in L^{2}\left(P^{\prime}\right)$ to our initial stochastic basis and represent $f(B, L)$ on $\left(\Omega, P, \mathcal{F},\left\{\mathcal{F}_{t}\right\}_{0 \leq t \leq T}, B, L\right)$ as

$$
E_{P^{\prime}}\left[Y^{\prime} J_{T}^{\prime \diamond} M_{T}^{\prime \diamond}\right]
$$

where

$$
\begin{gathered}
J_{T}^{\prime \diamond}=\exp ^{\diamond}\left(\int_{0}^{T} \int_{\mathbb{R}_{0}} \log ^{\diamond}(1+\dot{\widetilde{N}}(\omega, s, x)) N^{\prime}\left(\omega^{\prime}, d s, d x\right)-\int_{0}^{T} \int_{\mathbb{R}_{0}} \dot{\widetilde{N}}(\omega, s, x) \nu(d x) d s\right) \\
M_{T}^{\prime \diamond}=\exp ^{\diamond}\left(\int_{0}^{T} \dot{B}_{u}(\omega) d B_{u}^{\prime}-\frac{1}{2} \int_{0}^{T}\left(\dot{B}_{u}(\omega)\right)^{\diamond 2} d u\right) .
\end{gathered}
$$

Lemma 5.4 follows from the fact that the Fourier coefficients of (5.3) and (5.5) are the same. In passing we can note that Lemma 5.4 extends immediately a well known result for continuous SDE's (see f.ex. [?]): In the above notation let $f$ be the function such that a square integrable strong solution of (5.2) is given by $f(B, L)$. Then for the same function $f$ the functional $f\left(B^{\prime}, L^{\prime}\right)$ is a strong solution corresponding to the basis $\left(\Omega^{\prime}, P^{\prime}, \mathcal{F}^{\prime},\left\{\mathcal{F}_{t}^{\prime}\right\}_{0 \leq t \leq T}, B^{\prime}, L^{\prime}\right)$.

5.1. A convergence theorem for SDE's with irregular coefficients. Let $b, b_{n}: \mathbb{R} \longrightarrow \mathbb{R}, n=1,2, \ldots$, be measurable functions such that

$$
b_{n}(y) \rightarrow b(y)
$$

almost everywhere (denoted a.e.) with respect to the Lebesgue measure and

$$
E_{\mu}^{y}\left[\exp \left(\int_{0}^{T} \sup _{n} b_{n}^{2}\left(\widehat{B}_{t}\right) d t\right)\right]<\infty
$$

We assume that for $b_{n}, n=1,2, \ldots$, the corresponding distorted Brownian motion (5.1) admits a strong solution $Y_{t}^{b_{n}}$ which has the representation of Theorem 3.8

$$
Y_{t}^{b_{n}}=E_{\widehat{\mu}}^{y}\left[\widehat{B}_{t} \exp ^{\diamond}\left(\int_{0}^{T}\left(\dot{B}_{u}+b_{n}\left(\widehat{B}_{u}\right)\right) d \widehat{B}_{u}-\frac{1}{2} \int_{0}^{T}\left(\dot{B}_{u}+b_{n}\left(\widehat{B}_{u}\right)\right)^{\diamond 2} d u\right)\right] .
$$

Lemma 5.5. Define

$$
Y_{t}:=E_{\widehat{\mu}}^{y}\left[\widehat{B}_{t} \exp ^{\diamond}\left(\int_{0}^{T}\left(\dot{B}_{u}+b\left(\widehat{B}_{u}\right)\right) d \widehat{B}_{u}-\frac{1}{2} \int_{0}^{T}\left(\dot{B}_{u}+b\left(\widehat{B}_{u}\right)\right)^{\diamond 2} d u\right)\right] .
$$

Then $Y_{t}$ is an element in the Hida distribution space.

Proof. It is sufficient to show that

$$
E_{\widehat{\mu}}^{y}\left[\sup _{z \in \mathbb{K}_{q}(R)}\left|\widehat{B}_{t} \exp \left(\int_{0}^{T} \phi_{z}(u)+b\left(\widehat{B}_{u}\right) d \widehat{B}_{u}-\frac{1}{2} \int_{0}^{T}\left(\phi_{z}(u)+b\left(\widehat{B}_{u}\right)\right)^{2} d u\right)\right|\right]<\infty
$$


for some $q, R$, where $\phi_{z}(s)=\mathcal{H}\left(\dot{B}_{u}\right)(z)$. Because of condition (5.7) we can define the probability measure $\frac{d \mu^{*}}{d \widehat{\mu}}=\mathcal{E}\left(\int_{0}^{t} b\left(\widehat{B}_{u}\right) d \widehat{B}_{u}\right)$ and we get

$$
\begin{aligned}
& E_{\widehat{\mu}}^{y}\left[\sup _{z \in \mathbb{K}_{q}(R)}\left|\widehat{B}_{t} \exp \left(\int_{0}^{T} \phi_{z}(u)+b\left(\widehat{B}_{u}\right) d \widehat{B}_{u}-\frac{1}{2} \int_{0}^{T}\left(\phi_{z}(u)+b\left(\widehat{B}_{u}\right)\right)^{2} d u\right)\right|\right] \\
& =E_{\mu^{*}}^{y}\left[\left|\widehat{B}_{t}\right| \sup _{z \in \mathbb{K}_{q}(R)}\left|\exp \left(\int_{0}^{T} \phi_{z}(u) d B_{u}^{*}-\frac{1}{2} \int_{0}^{T} \phi_{z}^{2}(u) d u\right)\right|\right] \\
& \leq \text { const. } E_{\mu^{*}}^{y}\left[\sup _{z \in \mathbb{K}_{q}(R)}\left|\exp \left(\int_{0}^{T} 2 \phi_{z}(u) d B_{u}^{*}-\int_{0}^{T} \phi_{z}^{2}(u) d u\right)\right|\right]^{\frac{1}{2}}
\end{aligned}
$$

where $B_{u}^{*}=\widehat{B}_{t}-b\left(\widehat{B}_{t}\right)$ is Brownian motion under $\mu^{*}$. For the last inequality we used Hölder and the fact that by condition (5.7) $\widehat{B}_{t}$ is square integrable under $\mu^{*}$. Further, it can be proven with the help of Fernique that

$$
E_{\mu^{*}}^{y}\left[\sup _{z \in \mathbb{K}_{q}(R)}\left|\exp \int_{0}^{T} 4 \phi_{z}(u) d B_{u}^{*}\right|\right]<\infty
$$

for some $q, R$ (see Lemma 3.1 in [15]).We thus get by using Hölder again

$$
\begin{aligned}
& E_{\mu^{*}}^{y}\left[\sup _{z \in \mathbb{K}_{q}(R)}\left|\exp \left(\int_{0}^{T} 2 \phi_{z}(u) d B_{u}^{*}-\int_{0}^{T} \phi_{z}^{2}(u) d u\right)\right|\right] \\
& \leq E_{\mu^{*}}^{y}\left[\sup _{z \in \mathbb{K}_{q}(R)}\left|\exp \int_{0}^{T} 4 \phi_{z}(u) d B_{u}^{*}\right|\right]^{\frac{1}{2}} E_{\mu^{*}}^{y}\left[\sup _{z \in \mathbb{K}_{q}(R)}\left|\exp \int_{0}^{T} 2 \phi_{z}^{2}(u) d u\right|\right]^{\frac{1}{2}} \leq \infty .
\end{aligned}
$$

The result follows.

Lemma 5.6. Suppose that the integrability condition (5.7) holds. Then $Y_{t}$ given by (5.9) is square integrable for all $t$.

Proof. Note that by property (5.3), property (5.4) and the calculations in the last proof we get

$$
\begin{aligned}
& \left(E_{\widehat{\mu}}^{y}\left[\widehat{B}_{t} \exp ^{\diamond}\left(\int_{0}^{T}\left(\dot{B}_{u}+b\left(\widehat{B}_{u}\right)\right) d \widehat{B}_{u}-\frac{1}{2} \int_{0}^{T}\left(\dot{B}_{u}+b\left(\widehat{B}_{u}\right)\right)^{\diamond 2} d u\right)\right]\right)^{2} \\
& =\left(E_{\mu^{*}}^{y}\left[\widehat{B}_{t} \exp ^{\diamond}\left(\int_{0}^{t} \dot{B}_{u} d B_{u}^{*}-\frac{1}{2} \int_{0}^{t}\left(\dot{B}_{u}\right)^{\diamond 2} d u\right)\right]\right)^{2} \\
& =\left(E_{\mu^{*}}^{y}\left[E\left[\widehat{B}_{t} \mid \mathcal{F}_{t}^{*}\right] \exp ^{\diamond}\left(\int_{0}^{t} \dot{B}_{u} d B_{u}^{*}-\frac{1}{2} \int_{0}^{t}\left(\dot{B}_{u}\right)^{\diamond 2} d u\right)\right]\right)^{2} \\
& =E_{\mu^{*}}^{y}\left[\left(E\left[\widehat{B}_{t} \mid \mathcal{F}_{t}^{*}\right]\right)^{2} \exp ^{\diamond}\left(\int_{0}^{t} \dot{B}_{u} d B_{u}^{*}-\frac{1}{2} \int_{0}^{t}\left(\dot{B}_{u}\right)^{\diamond 2} d u\right)\right] \\
& \leq E_{\mu^{*}}^{y}\left[\left(\widehat{B}_{t}\right)^{2} \exp ^{\diamond}\left(\int_{0}^{t} \dot{B}_{u} d B_{u}^{*}-\frac{1}{2} \int_{0}^{t}\left(\dot{B}_{u}\right)^{\diamond 2} d u\right)\right]
\end{aligned}
$$


where $\mathcal{F}_{t}^{*}$ denotes the filtration generated by $B^{*}$. By property (5.5) the object in (5.10) is a 'lifting' of a square integrable random variable and the result follows.

With the help of the characterization of convergence in stochastic distribution spaces through the Hermite transform we are now ready to state the following convergence theorem for SDE's with irregular coefficients.

THEOREM 5.7. Assume $b$ and $b_{n}, n=1,2, \ldots$, fulfill conditions (5.6) and (5.7). If there exists a subsequence $Y_{t}^{b_{n_{j}}}, j=1,2, \ldots$, that converges in probability for all $t \in[0, T]$, then there exists a unique square integrable strong solution of (5.1) with coefficient $b$ given through $Y_{t}$ in (5.9). Conversely, assume there exists a square integrable strong solution of (5.1) with coefficient $b$, then this solution is uniquely given through $Y_{t}$ in (5.9) and there exists a subsequence $Y_{t}^{b_{n_{j}}}, j=1,2, \ldots$, converging to this solution in $L^{2}(\mu)$ for all $t \in[0, T]$.

Proof. First we verify that $\mathcal{H}\left(Y_{t}^{b_{n}}\right)(z) \rightarrow \mathcal{H}\left(Y_{t}\right)(z)$ for $n \longrightarrow \infty$ pointwise boundedly for $z \in \mathbb{C}_{c}^{\mathbb{N}}$. Some calculations including Hölder give

$$
\begin{aligned}
& \left|\mathcal{H}\left(Y_{t}\right)(z)-\mathcal{H}\left(Y_{t}^{b_{n}}\right)(z)\right| \\
& =\mid E_{\widehat{\mu}}^{y}\left[\widehat{B}_{t} \exp \left(\int_{0}^{T}\left(\phi_{z}(u)+b\left(\widehat{B}_{u}\right)\right) d \widehat{B}_{u}-\frac{1}{2} \int_{0}^{T}\left(\phi_{z}(u)+b\left(\widehat{B}_{u}\right)\right)^{2} d u\right)\right] \\
& -E_{\widehat{\mu}}^{y}\left[\widehat{B}_{t} \exp \left(\int_{0}^{T}\left(\phi_{z}(u)+b_{n}\left(\widehat{B}_{u}\right)\right) d \widehat{B}_{u}-\frac{1}{2} \int_{0}^{T}\left(\phi_{z}(u)+b_{n}\left(\widehat{B}_{u}\right)\right)^{2} d u\right)\right] \mid \\
& \leq\left\{\text { const. } E_{\widehat{\mu}}^{y}\left[\left(\int_{0}^{T}\left(b\left(\widehat{B}_{u}\right)-b_{n}\left(\widehat{B}_{u}\right)\right) d \widehat{B}_{u}\right)^{2}\right]\right. \\
& \text { +const. } \left.E_{\widehat{\mu}}^{y}\left[\left(\int_{0}^{T}\left|\left(\phi_{z}(u)+b\left(\widehat{B}_{u}\right)\right)^{2}-\left(\phi_{z}(u)+b_{n}\left(\widehat{B}_{u}\right)\right)^{2}\right| d u\right)^{2}\right]\right\}^{\frac{1}{2}} \\
& \cdot \sup _{n} E_{\widehat{\mu}}^{y}\left[\widehat{B}_{t}^{2}\left|\exp \left(\int_{0}^{T} 2\left(\phi_{z}(u)+b_{n}\left(\widehat{B}_{u}\right)\right) d \widehat{B}_{u}-\int_{0}^{T}\left(\phi_{z}(u)+b_{n}\left(\widehat{B}_{u}\right)\right)^{2} d u\right)\right|\right]^{\frac{1}{2}} .
\end{aligned}
$$

By condition (5.7) and calculations similar to the ones done in the proof of Lemma 5.5 one sees that the last factor is bounded. Then, using the fact that Brownian motion does not stay in a given set of Lebesgue measure zero for a.a. $u \in[0, T]$ and dominated convergence on the first factor yields the desired convergence of Hermite transforms.

Now assume w.l.o.g. $Y_{t}^{b_{n}}, n=1,2, \ldots$, converges in probability for all $t \in[0, T]$. Because $\mathcal{H}\left(Y_{t}^{b_{n}}\right)(z) \rightarrow \mathcal{H}\left(Y_{t}\right)(z)$ the limit in probability must be $Y_{t}$. Given $\varphi \in C_{b}(\mathbb{R})$ we find in the same way as above

$$
\begin{aligned}
& \mathcal{H}\left(\varphi\left(Y_{t}^{b_{n}}\right)\right)(z) \\
& =E_{\widehat{\mu}}^{y}\left[\varphi\left(\widehat{B}_{t}\right) \exp \left(\int_{0}^{T} \phi_{z}(u)+b_{n}\left(\widehat{B}_{u}\right) d \widehat{B}_{u}-\frac{1}{2} \int_{0}^{T}\left(\phi_{z}(u)+b_{n}\left(\widehat{B}_{u}\right)\right)^{2} d u\right)\right] \\
& \longrightarrow E_{\widehat{\mu}}^{y}\left[\varphi\left(\widehat{B}_{t}\right) \exp \left(\int_{0}^{T} \phi_{z}(u)+b\left(\widehat{B}_{u}\right) d \widehat{B}_{u}-\frac{1}{2} \int_{0}^{T}\left(\phi_{z}(u)+b\left(\widehat{B}_{u}\right)\right)^{2} d u\right)\right]
\end{aligned}
$$


for $n \longrightarrow \infty$, for all $z \in \mathbb{C}_{c}^{\mathbb{N}}$. But we also find because of convergence in probability (by possibly taking a subsequence)

$$
\mathcal{H}\left(\varphi\left(Y_{t}^{b_{n}}\right)\right)(z) \longrightarrow \mathcal{H}\left(\varphi\left(Y_{t}\right)\right)(z) \text { for } n \longrightarrow \infty
$$

This shows the transformation property (5.4) is valid for $Y_{t}$ with $\varphi \in C_{b}(\mathbb{R})$ and by an approximation argument we get for all $\varphi: \mathbb{R} \rightarrow \mathbb{R}$ such that $\varphi\left(Y_{t}\right) \in L^{2}(\mu)$ that

$$
\varphi\left(Y_{t}\right)=E_{\widehat{\mu}}^{y}\left[\varphi\left(\widehat{B}_{t}\right) \exp ^{\diamond}\left(\int_{0}^{T}\left(\dot{B}_{u}+b\left(\widehat{B}_{u}\right)\right) d \widehat{B}_{u}-\frac{1}{2} \int_{0}^{T}\left(\dot{B}_{u}+b\left(\widehat{B}_{u}\right)\right)^{\diamond 2} d u\right)\right] .
$$

We proceed to prove that $Y_{t}$ actually solves (5.1). For this purpose we need to have process $Y_{t}$ has a continuous modification. One easily sees that property (5.4) carries over to increments of the type $Y_{t}-Y_{s}, 0 \leq s, t \leq T$. In particular we get for $\varphi(y)=y^{2}$ the relation

$$
\begin{aligned}
& \left(Y_{t}-Y_{s}\right)^{2} \\
& =E_{\widehat{\mu}}^{y}\left[\left(\widehat{B}_{t}-\widehat{B}_{s}\right)^{2} \exp ^{\diamond}\left(\int_{0}^{T} \dot{B}_{u}+b\left(\widehat{B}_{u}\right) d \widehat{B}_{u}-\frac{1}{2} \int_{0}^{T}\left(\dot{B}_{u}+b\left(\widehat{B}_{u}\right)\right)^{\diamond 2} d u\right)\right] .
\end{aligned}
$$

The latter leads to

$$
\begin{aligned}
E_{\mu}^{y}\left[\left(Y_{t}-Y_{s}\right)^{2}\right] & =E_{\widehat{\mu}}^{y}\left[\left(\widehat{B}_{t}-\widehat{B}_{s}\right)^{2} \exp \left(\int_{0}^{T} b\left(\widehat{B}_{u}\right) d \widehat{B}_{u}-\frac{1}{2} \int_{0}^{T} b^{2}\left(\widehat{B}_{u}\right) d u\right)\right] \\
& \leq \text { const. }|t-s|^{2}
\end{aligned}
$$

Then Kolmogorov's Lemma provides a continuous modification of $Y_{t}$. For a moment let $z \in \mathbb{R}_{c}^{\mathbb{N}}$. Since $\widehat{B}_{t}$ is a weak solution of $(5.1)$ for the drift $b(y)+\phi_{z}(t)$ with respect to the measure $d \mu^{*}=\mathcal{E}\left(\int_{0}^{T}\left(\phi_{z}(u)+b\left(\widehat{B}_{u}\right)\right) d \widehat{B}_{u}\right) d \mu$ we obtain that

$$
\begin{aligned}
\mathcal{H}\left(Y_{t}\right)(z) & E_{\widehat{\mu}}^{y}\left[\widehat{B}_{t} \exp \left(\int_{0}^{T} \phi_{z}(u)+b\left(\widehat{B}_{u}\right) d \widehat{B}_{u}-\frac{1}{2} \int_{0}^{T}\left(\phi_{z}(u)+b\left(\widehat{B}_{u}\right)\right)^{2} d u\right)\right] \\
= & E_{\mu^{*}}^{y}\left[\widehat{B}_{t}\right]=E_{\mu^{*}}^{y}\left[\int_{0}^{T} \phi_{z}(u)+b\left(\widehat{B}_{u}\right) d u\right] \\
= & \int_{0}^{t} E_{\widehat{\mu}}^{y}\left[b\left(\widehat{B}_{u}\right) \exp \left(\int_{0}^{T} \phi_{z}(u)+b\left(\widehat{B}_{u}\right) d \widehat{B}_{u}-\frac{1}{2} \int_{0}^{T}\left(\phi_{z}(u)+b\left(\widehat{B}_{u}\right)\right)^{2} d u\right)\right] d u \\
& +\mathcal{H}\left(B_{t}\right)(z) .
\end{aligned}
$$

So by assumption and (5.11) applied to $b$ it follows that

$$
\mathcal{H}\left(Y_{t}\right)(z)=\mathcal{H}\left(\int_{0}^{t} b\left(Y_{u}\right) d u\right)(z)+\mathcal{H}\left(B_{t}\right)(z)
$$

Since both sides of the last relation have analytical extensions to $z \in \mathbb{C}_{c}^{\mathbb{N}}$ we see that

$$
Y_{t}=\int_{0}^{t} b\left(Y_{u}\right) d u+B_{t}
$$


One sees easily that a solution of (5.1) is unique in law (see e.g. Prop. 5.3.10 in [13]). Then $Y_{t}$ is a unique strong solution which by Theorem 3.8 takes the form (5.9).

Conversely, assume there exists a strong solution. Again by uniqueness in law and Theorem 3.8 this solution must be given by $Y_{t}$. Using Hölder and condition (5.7) it is easy to see that

$$
\left\|Y_{t}^{b_{n}}\right\|_{L^{2}(\mu)}^{2} \leq M<\infty
$$

as well as

$$
\left\|\left(Y_{t}^{b_{n}}\right)^{2}\right\|_{L^{2}(\mu)}^{2} \leq M<\infty .
$$

Thus the sequences $Y_{t}^{b_{n}}$ as well as $\left(Y_{t}^{b_{n}}\right)^{2}, n=1,2, \ldots$, are relatively compact in $L^{2}(\mu)$ in the weak sense. This implies that there exists a subsequence of $Y_{t}^{b_{n_{j}}}$ and $\left(Y_{t}^{b_{n_{j}}}\right)^{2}$ which converges to elements $Z_{t}, Z_{t}^{\prime} \in L^{2}(\mu)$ weakly. By the convergence of Hermite transforms we must have

$$
\begin{aligned}
& Z_{t}=Y_{t}=E_{\widehat{\mu}}^{y}\left[\widehat{B}_{t} \exp ^{\diamond}\left(\int_{0}^{T}\left(\dot{B}_{u}+b\left(\widehat{B}_{u}\right)\right) d \widehat{B}_{u}-\frac{1}{2} \int_{0}^{T}\left(\dot{B}_{u}+b\left(\widehat{B}_{u}\right)\right)^{\diamond 2} d u\right)\right] \\
& Z_{t}^{\prime}=E_{\widehat{\mu}}^{y}\left[\left(\widehat{B}_{t}\right)^{2} \exp ^{\diamond}\left(\int_{0}^{T}\left(\dot{B}_{u}+b\left(\widehat{B}_{u}\right)\right) d \widehat{B}_{u}-\frac{1}{2} \int_{0}^{T}\left(\dot{B}_{u}+b\left(\widehat{B}_{u}\right)\right)^{\diamond 2} d u\right)\right] .
\end{aligned}
$$

However, since property $(5.11)$ is valid for $Y_{t}$ we see that $Z_{t}^{\prime}=\left(Z_{t}\right)^{2}$. This implies $L^{2}(\mu)$ convergence of a subsequence as desired.

REMARK 5.8. For equation (5.2) including jumps one sees from the last proof that a corresponding result can be obtained by supposing sufficient integrability on $\mathcal{H}\left(J_{T}^{\diamond}\right)(z)$ where $J_{T}^{\diamond}$ is given in (3.15). However, we need in addition to have the coefficients $\gamma_{n}(y) \rightarrow \gamma(y)$ for all $y$, and not only a.a $y$, because we are dealing with finite variation jump processes.

5.2. A sufficient criteria for strong solutions. In this subsection we want to apply Theorem 5.7 in order to state a sufficient criteria for a strong solution of SDE (5.1). Furthermore we formulate a comparison result for SDE's with irregular coefficients. We recall that the results go through also for time inhomogeneous coefficients. The main achievment of this Subsection is formulated as follows:

THEOREM 5.9. Let us require that for $b: \mathbb{R} \longrightarrow \mathbb{R}$ the integrability condition

$$
E_{\mu}^{y}\left[\exp \left(\int_{0}^{T} b^{2}\left(\widehat{B}_{t}\right) d t\right)\right]<\infty
$$

holds. Then there exists a unique square integrable strong solution $Y_{t}$ of (5.1), which is explicitly given by (5.9).

Further, if $b_{1}, b_{2}: \mathbb{R} \longrightarrow \mathbb{R}$ are two coefficients for which (5.12) holds and such that $b_{1}(y) \leq b_{2}(y)$ for $y \in \mathbb{R}$, then the corresponding strong solutions $Y_{t}^{b_{1}}, Y_{t}^{b_{2}}$ fulfill

$$
Y_{t}^{b_{1}}(\omega) \leq Y_{t}^{b_{2}}(\omega)
$$


almost surely for all $t \in[0, T]$.

We divide the proof of Theorem 5.9 into several help lemmas.

LEMmA 5.10. Let $\varphi: \mathbb{R} \longrightarrow \mathbb{R}$ be a lower semicontinuous bounded function. Then there is a sequence of bounded increasing Lipschitz functions $\varphi_{n}: \mathbb{R} \longrightarrow \mathbb{R}$ such that $\varphi_{n}(y) \nearrow \varphi(y)$ for all $y \in \mathbb{R}$.

Proof. Let us define the sequence of functions

$$
\varphi_{n}(x)=\inf _{p \in \mathbb{R}}\{\varphi(p)+n|x-p|\} .
$$

It is clear from the definition that $\varphi_{n}(x) \nearrow$ for all $x$ and that $\varphi_{n} \leq \varphi$. Now for $\varepsilon>0$ choose $p_{\varepsilon}$ such that

$$
\begin{aligned}
\varphi_{n}(x) & \geq \varphi\left(p_{\varepsilon}\right)+n\left|x-p_{\varepsilon}\right|-\varepsilon \\
& \geq \varphi\left(p_{\varepsilon}\right)+n\left|y-p_{\varepsilon}\right|+n\left|x-p_{\varepsilon}\right|-n\left|y-p_{\varepsilon}\right|-\varepsilon \\
& \geq \varphi\left(p_{\varepsilon}\right)+n\left|y-p_{\varepsilon}\right|-n|x-y|-\varepsilon \\
& \geq \varphi_{n}(y)-n|x-y|-\varepsilon .
\end{aligned}
$$

So by interchanging the roles of $x$ and $y$ we obtain that

$$
\left|\varphi_{n}(x)-\varphi_{n}(y)\right| \leq n|x-y| .
$$

In order to prove the convergence of $\varphi_{n}$ we take for every $n \in \mathbb{N}$ a $p_{n}$ such that

$$
\varphi(x) \geq \varphi_{n}(x) \geq \varphi\left(p_{n}\right)+n\left|x-p_{n}\right|-\frac{1}{n} .
$$

Since $\varphi$ is bounded we deduce that

$$
p_{n} \longrightarrow x .
$$

Because of

$$
\varphi(x) \leq \lim \inf _{n \longrightarrow \infty} \varphi\left(p_{n}\right)
$$

and

$$
\varphi(x) \geq \varphi_{n}(x) \geq \varphi\left(p_{n}\right)-\frac{1}{n}
$$

it follows that

$$
\varphi_{n}(x) \nearrow \varphi(x) \text { for all } x,
$$

which was the result to prove.

LEMmA 5.11. Suppose $b_{1}, b_{2}: \mathbb{R} \longrightarrow \mathbb{R}$ are both lower semicontinuous or both upper semicontinuous functions such that $b_{1}(y) \leq b_{2}(y)$ for $y \in \mathbb{R}$. Further assume that (5.12) holds for $b_{i}, i=1,2$. Then $Y_{t}^{b_{1}}, Y_{t}^{b_{2}}$ given by (5.8) are strong solutions of equation (5.1) and

$$
Y_{t}^{b_{1}}(\omega) \leq Y_{t}^{b_{2}}(\omega)
$$


almost surely for all $t \in[0, T]$.

Proof. Consider first the case when $b_{1}, b_{2}$ are lower semicontinuous bounded functions. By Lemma 5.10 we have Lipschitz functions $g_{n}$ such that $g_{n} / b_{1}$ for all $x$. For the coefficients $g_{n}$ we know there exist corresponding strong solutions $Y_{t}^{g_{n}}$. By the usual comparison theorem (see e.g. [13]) and Theorem 5.7 we have $Y_{t}^{g_{n}} \nearrow Y_{t}^{b_{1}}$ in $L^{2}(\mu)$ as $n \rightarrow \infty$ (at least for a subsequence). Also Theorem 5.7 tells us that $b_{1}$ admits a strong solution $Y_{t}^{b_{1}}$. In the same way we get a strong solutions $Y_{t}^{b_{2}}$. Further, since $Y_{t}^{g_{n}} \leq Y_{t}^{b_{2}}$ for all $n$ and for a subsequence $Y_{t}^{g_{n}} \nearrow Y_{t}^{b_{1}}$ a.s it follows that $Y_{t}^{b_{1}} \leq Y_{t}^{b_{2}}$ a.s..

For general unbounded lower semicontinuous functions $b_{1}, b_{2}$ we can use truncation and the same arguments as before, now on a sequence of increasing (decreasing) lower semicontinuous bounded coefficients, in order to obtain the result.

If now $u_{1}, u_{2}$ are upper semicontinuous functions such that condition (5.12) holds as well as $u_{1} \leq u_{2}$, then by definition we observe that $b_{2}(x):=-u_{1}(-x)$ and $b_{1}(x):=$ $-u_{2}(-x)$ are lower semicontinuous functions satisfying (5.12) and $b_{2}(x) \geq b_{1}(x)$. We know from before that there exist unique strong solutions $Y_{t}^{(1)}, Y_{t}^{(2)}$ such that

$$
d Y_{t}^{(i)}=b_{i}\left(Y_{t}^{(i)}\right) d t-d B_{t}, i=1,2
$$

and

$$
Y_{t}^{(1)}(\omega) \leq Y_{t}^{(2)}(\omega) \text { a.s.. }
$$

Thus $X_{t}^{(1)}:=-Y_{t}^{(2)}, X_{t}^{(2)}:=-Y_{t}^{(1)}$ are the corrresponding strong solutions of 5.1 for $u_{1}, u_{2}$ such that

$$
X_{t}^{(1)}(\omega) \leq X_{t}^{(2)}(\omega) \text { a.s. }
$$

We can now proceed to prove Theorem 5.9:

Proof. (Theorem 5.9) Assume first that $b$ is bounded. By virtue of the theorem of Vitali-Caratheodory (see e.g. [25]) there exists a sequence of (bounded) upper semicontinuous functions $u_{n}$ such that

$$
u_{n} \leq b
$$

and

$$
u_{n}(x) \longrightarrow b(x) \text { a.e. }
$$

(at least for a subsequence). Define the functions

$$
b_{n}=\sup _{k=1}^{n} u_{k} .
$$

Since the supremum of a finite number of upper semicontinuous functions is upper semicontinuous we see that $b_{n}$ enjoys the same property and

$$
b_{n} \nearrow b \text { a.e. }
$$

Thus with the help of Theorem 5.11 and Theorem 5.7 we conclude with similar arguments as in the proof of Theorem 5.11 that $b$ admits a unique strong solution. Also 
for two coefficients $b_{1}, b_{2}: \mathbb{R} \longrightarrow \mathbb{R}$ such that $b_{1}(y) \leq b_{2}(y)$ for $y \in \mathbb{R}$ we get by similar arguments as in the proof of Theorem 5.11 that for all $t \in[0, T]$

$$
Y_{t}^{b_{1}}(\omega) \leq Y_{t}^{b_{2}}(\omega) \text { a.s.. }
$$

For general unbounded coefficients $b$ the result follows by truncation and the same type of arguments as above applied to sequences of monotonic growing bounded coefficients.

Using Theorem 5.9 we give a deterministic integrability condition on the drift which assures the existence of a unique strong solution of (5.1).

TheOrem 5.12. Assume that

$$
\int_{N}^{\infty} b^{2}(y) d y<\infty
$$

for all $N \in \mathbb{R}$ as well as

$$
b(y)=O(1) \text { for } y \longrightarrow-\infty .
$$

Then there exists a unique strong solution $Y_{t}$ which has the explicit representation (5.9).

For the proof of Theorem 5.12 we resort to the following result of Yor and Salminen $[26]$ :

LEMMA 5.13. Let $f$ be a non-negative measurable function which satisfies the assumptions of Theorem 5.12. Denote by $B_{t}^{(\nu)}=B_{t}+\nu t$ a Brownian motion with drift $\nu>0$ and set $I_{\infty}(f)=\int_{0}^{\infty} f\left(B_{s}^{(\nu)}\right) d s$. Then there exists a $\gamma>0$ such that for all $y$

$$
E^{y}\left[\exp \left(\gamma I_{\infty}(f)\right)\right]<\infty .
$$

In particular, if $f(y) \leq C_{N^{*}}$ for all $y \leq N^{*}, N^{*} \in \mathbb{R}$, then (5.13) is valid for

$$
\gamma<\min \left\{\frac{\nu}{2 \int_{N^{*}}^{\infty} f(y) d y}, \frac{\nu^{2}}{4 C_{N^{*}}}\right\} .
$$

Proof. (Theorem 5.12) By Lemma 5.13 we obtain that

$$
\left.E_{\mu}^{y}\left[\exp \left(\gamma \int_{0}^{T} b^{2}\left(B_{s}^{(\nu)}\right) d s\right)\right)\right]<\infty
$$

for a $\gamma>0$ and all $y$. W.l.o.g. let $y=0$. It follows from Girsanov's theorem that $B_{t}-\nu t$ is a Brownian motion with respect to the measure $\bar{\mu}=\mathcal{E}\left(\nu B_{T}\right) d \mu$. Thus we get

$$
\begin{aligned}
& E_{\mu}^{y}\left[\exp \left(\int_{0}^{T} b^{2}\left(B_{s}\right) d s\right)\right] \\
= & E_{\bar{\mu}}^{y}\left[\exp \left(\int_{0}^{T} b^{2}\left(\widehat{B}_{s}+\nu s\right) d s\right) \mathcal{E}\left(-\nu B_{T}\right)\right] \\
= & E_{\bar{\mu}}^{y}\left[\exp \left(\int_{0}^{T} b^{2}\left(\widehat{B}_{s}+\nu s\right) d s\right) \mathcal{E}\left(-\nu\left(\widehat{B}_{T}+\nu T\right)\right)\right] \\
\leq & E_{\bar{\mu}}^{y}\left[\exp \left(2 \int_{0}^{T} b^{2}\left(\widehat{B}_{s}+\nu s\right) d s\right)\right]^{\frac{1}{2}} E_{\bar{\mu}}^{y}\left[\mathcal{E}^{2}\left(-\nu\left(\widehat{B}_{T}+\nu T\right)\right)\right]^{\frac{1}{2}} \\
< & \infty, \text { if } \gamma>2 .
\end{aligned}
$$


Because of relation (5.14) we can choose $\nu>0$ large enough such that $\gamma>2$. This shows that (5.12) is fulfilled. So the result follows from Theorem 5.9.

Example 5.14. Consider the drift

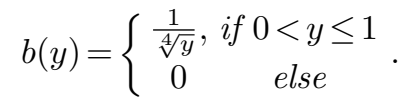

Then $b$ satisfies the conditions of Theorem 5.4. Thus

$$
d Y_{t}=\chi_{(0,1]}\left(Y_{t}\right) \frac{1}{\sqrt[4]{Y_{t}}} d t+d B_{t}
$$

has a unique strong solution.

REMARK 5.15. In Theorem 5.9 we presented an alternative proof to a result of Zvonkin [35], where the special case of bounded drift coefficients were treated. Let us mention that Zvonkin was the first, who studied strong solutions of SDE's with irregular coefficients. We also remark that our result is novel. To the best of our knowledge this result provides the weakest known integrability condition on the (time homogeneous) drift coefficient to elicit unique strong solutions. For similar results we refer to the recent papers [14], [7], where the authors require a higher order of integrability on the drift.

REMARK 5.16. Concerning the Lévy-Itô diffusion including jumps we point out that we can attain a similar result to Theorem 5.9 by applying comparison results for jump diffusions ([24]) just as in the above arguments. However since comparison theorems for jump SDE's are more restrictive we are only able to capture the case of coefficients $\gamma$ in $(5.2)$, which are piecewise monotone.

REMARK 5.17. A central step in the proof of Theorem 5.9 was the approximation of the a priori distributional object $Y_{t}$ in (5.9) by strong solutions of (5.1). We used the (almost everywhere) convergence of the strong solutions to $Y_{t}$ to deduce the transformation property (5.4). The latter property was essential for the verification of $Y_{t}$ as a strong solution of (5.1). At the moment we are not able to extend the results of this Subsection to the multidimensional or even infinite dimensional case since our proofs heavily rely on comparison results for SDE's. To overcome this deficiency it is e.g. conceivable to exploit the Skorohod embedding technique to achieve a.econvergence.

Acknowledgements. We thank F. E. Benth for helpful comments and discussions.

\section{REFERENCES}

[1] R. F. Bass, Stochastic differential equations driven by symmetric stable processes, Séminaire de Probabilités, to appear, 2003.

[2] R. F. Bass, K. Burdzy and Z-Q Chen, Stochastic differential equations driven by stable processes for which pathwise uniqueness fails, University of Washington, Seattle, Preprint, 2003.

[3] J. Bertoin, Lévy Processes, Cambridge University Press, Cambridge 1996.

[4] A. S. Cherny, On the strong and weak solutions of stohastic differential equations governing Bessel processes, Stochastics and Stochastics Reports, 70, 3-4, 213-219.

[5] S. Fang and T. Zhang, Stochastic differential equations with non-Lipschitzian coefficients: I. Pathwise uniqueness and Large deviations, Preprint, University of Manchester, 2003. 
[6] I. Gyöngy and N. V. Krylov, Existence of strong solutions for Itô's stochastic equations via approximations, Probab, Theory Relat. Fields, 105, 143-158, 1996.

[7] I. Gyöngy and T. Martinez, On stochastic differential equations with locally unbounded drift, Czechoslovak Mathematical Journal, 51 (4), 763-783, 2001.

[8] T. Hida, H.-H. Kuo, J. Potthoff and J. Streit, White Noise, An Infinite Dimensional Approach, Kluwer, 1993.

[9] H. Holden, B. Øksendal, J. Ubøe and T.-S. Zhang, Stochastic Partial Differential Equations- A Modeling, White Noise Functional Approach, Birkhäuser, Boston, 1996.

[10] J. Jacod and A. N. Shiryaev, Limit Theorems for Stochastic Processes, Springer, Berlin Heidelberg New York, 1987.

[11] H. H. Kuo, White Noise Distribution Theory, Prob. and Soch. Series, Boca Raton, FL, CRC Press, 1996

[12] G. Kallianpur and J. Xiong, Stochastic differential equations in infinite dimensional spaces, IMS Lecture Notes-Monograph Series, 26, 1995.

[13] I. Karatzas and S. E. Shreve, Brownian Motion and Stochastic Calculus, secnded., SpringerVerlag.

[14] N. V. Krylov and M. Röckner, Strong solutions of stochastic equations with singular time dependent drift, to appear in Prob. Theory Rel. Fields, 2005.

[15] A. Lanconelli and F. Proske, On explicit strong solutions of Itô-SDE's and the Donsker delta function of a diffusion, Infin. Dimen. Anal. Quant. Prob. Related Topics, 7, 3, 2004.

[16] A. Lanconelli and F. Proske, Explicit strong solutions of stochastic differential equations on Hilbert spaces, Preprint series, University of Oslo, 34, 2003.

[17] A. Løkka and F. Proske, Infinite dimensional analysis of pure jump Lévy processes on Poisson space, to appear in Mathematica Scandinavica, 2005.

[18] A. Løkka, B. Øksendal and F. Proske, Stochastic partial differential equations driven by Lévy space time white noise, Annals of Appl. Prob., 14(3), 1506-1528, 2004.

[19] R. Mikulevicius and B. Rozovskii, Linear parabolic stochastic PDE's and Wiener chaos, SIAM J. Math. Anal. 29(2), 452-480, 1998

[20] N. Obata, White Noise Calculus and Fock Space, LNM 1577, Berlin, Springer-Verlag, 1994.

[21] B. Øksendal and F. Proske, White noise of Poisson random measures, Potential Analysis, 21(4), 375-403, 2004.

[22] E. Pardaux and S. Peng, Adapted solution of a backward stochastic differential equation, Systems and Control Letters, 14, 55-61, 1990.

[23] F. Proske, The general stochastic transport equation driven by Lévy white noise, Comm. in Math. Sci., 2(4), 627-641, 2004.

[24] S. Rong, Reflecting Stochastic Differential Equations with Jumps and Applications, Research Notes in Mathematics 408, Boca Raton: Chapman \& Hall/CRC, 2000.

[25] W. Rudin, Real and Complex Analysis, McGraw-Hill, 3rd edition, 1987.

[26] P. Salminen and M. Yor, On perpetual integral functionals of Brownian motion with drift, Preprint September 2003.

[27] K. Sato, Lévy Processes and Infinitely Divisible Distributions, Cambridge University Studies in Advanced Mathematics, Cambridge University Press, Cambridge, 68, 1999.

[28] H. J. Sussmann, On the gap between deterministic and stochastic ordinary equations, Ann. Prob., 6, 19-41, 1978.

[29] S. Thangavelu, Lectures on Hermite and Laguerre Functions, Math. Notes 42, Princeton University Press, 1993.

[30] A. Y. Veretennikov, On the strong solutions of stochastic differential equations, Theory Probab. Appl., 24, 354-366, 1979.

[31] A. Y. Veretennikov, On the strong solutions and explicit formulas for solutions of stochastic differential equations, Math USSR (Sbornik), 39, 387-403, 1981.

[32] A. Y. Veretennikov, On the criteria for existence of a strong solution to a stochastic equation Theory Probab. Appl., 27, 441-449, 1982.

[33] A. Y. Veretennikov and N. V. Krylov, On explicit formulas for solutions of stochastic differential equations, Math. USSR (Sbornik), 29, 229-256, 1976.

[34] D. R. Williams, Pathwise solutions of stochastic differential equations driven by Lévy processes, Rev. Mat. Iberoamericana 17, 295-329, 2001.

[35] A. K. Zvonkin, A transformation of the state space of a diffusion process that removes the drift, Math.USSR (Sbornik), 22, 129-149, 1974.

[36] A. K. Zvonkin and N. V. Krylov, On strong solutions of stochastic differential equations, Sel. Math. Sov., 19-61, 1981. 